\title{
Criminologie
}

\section{La réforme pénale au Canada : l'apport de la Commission canadienne sur la détermination de la peine}

\author{
Alvaro P. Pires
}

Volume 20, numéro 2, 1987

La détermination de la sentence

URI : https://id.erudit.org/iderudit/017250ar

DOI : https://doi.org/10.7202/017250ar

Aller au sommaire du numéro

Éditeur(s)

Les Presses de l'Université de Montréal

ISSN

0316-0041 (imprimé)

1492-1367 (numérique)

Découvrir la revue

Citer cet article

Pires, A. P. (1987). La réforme pénale au Canada : l’apport de la Commission canadienne sur la détermination de la peine. Criminologie, 20(2), 11-55. https://doi.org/10.7202/017250ar
Résumé de l'article

This study is a critical analysis of the penal reform project put forward by the Canadian Commission on Sentencing from the point of view of moderation. The author first considers the Commission's position in the penological debate between the Medical Model and the Justice Model. He then analyses the "new theory on sanctions" proposed by the Commission, as well as its position on "community measures" and on the ideal of rehabilitation. Finally, his analysis focuses on a number of the Commission's major recommendations for reform, its policy in terms of maximum and minimum sentences, the new "exceptional sentence" (intended to replace the present measures regarding "dangerous criminals"), its position on imprisonment of "twenty-five years' minimum" and its general guidelines for Canada. In conclusion, the author make an evaluation of the orientation of the Commission's reform with respect to the principle of moderation. 


\section{LA RÉFORME PÉNALE AU CANADA: \\ L'APPORT DE LA COMMISSION CANADIENNE SUR LA DÉTERMINATION DE LA PEINE Alvaro P. Pires*}

This study is a critical analysis of the penal reform project put forward by the Canadian Commission on Sentencing from the point of view of moderation. The author first considers the Commission's position in the penological debate between the Medical Model and the Justice Model. He then analyses the "new theory on sanctions" proposed by the Commission, as well as its position on "community measures" and on the ideal of rehabilitation. Finally, his analysis focuses on a number of the Commission's major recommendations for reform, its policy in terms of maximum and minimum sentences, the new "exceptional sentence (intended to replace the present measures regarding "dangerous criminals»), its position on imprisonment of «twenty-five years' minimum * and its general guidelines for Canada. In conclusion, the author make an evaluation of the orientation of the Commission's reform with respect to the principle of moderation.

La Commission canadienne sur la détermination de la peine (C.C.D.P.) vient de livrer au public son imposant projet de réforme. Ce rapport, que je n'hésite pas à qualifier d'ores et déjà de très important, mérite qu'on s'y attarde et qu'on l'étudie attentivement. Je suis convain$\mathrm{cu}$, quant à moi, qu'il marquera une étape dans le processus de réforme pénale canadien et dans l'évolution de notre pensée à cet égard.

Bien entendu, il est impossible de traiter d'un seul coup de tous les points qui réclament un débat, appellent une mise en valeur ou évoquent des idées complémentaires ou des contre-propositions. Pour s'en convaincre, il suffit de parcourir les quelque 600 pages qui le composent. Cette difficulté est d'autant plus grande qu'on ne saurait compter, à l'heure actuelle, sur une connaissance préalable du rapport chez la majorité des lecteurs. Il faut donc joindre la description à l'analyse.

Afin de donner une vision d'ensemble des principaux thèmes traités par la Commission, je commencerai donc par identifier les principales lignes de force du rapport (point 1.0.). Je préciserai alors le thème privilégié qui traverse tout l'article, en l'occurrence le principe de modération. Ensuite, je situerai dans ses grandes lignes l'orientation globale

* L'auteur est professeur au Département de criminologie de l'Université d'Ottawa. 
du rapport en termes de politique pénale (point 2.0.). J'examinerai par la suite la «théorie de la sentence» proposée par la Commission (point 3.0.), puis je passerai en revue quelques-unes des principales recommandations de réforme de la Commission, telles que sa politique en matière de "peines minimales et maximales», son modèle de lignes directrices, etc. (point 4.0). Je conclurai par une appréciation globale du projet de réforme eu égard au principe de modération (point 5.0.).

\subsection{LES CINQ FILS CONDUCTEURS DE LA C.C.D.P.}

À la lecture du rapport, nous pouvons identifier sans peine cinq fils conducteurs majeurs ou, si l'on veut, cinq objectifs principaux. Ces objectifs prennent tantôt la forme de véritables "problèmes à résoudre", tantôt celle de "buts à atteindre». Ceci est d'ailleurs facile à comprendre, car ces objectifs de réforme de la Commission sont nés de la reconnaissance de certains problèmes qui peuvent se rapporter, en partie du moins, au processus sentenciel. Ainsi, par exemple, si l'on reconnaît qu'il existe des formes injustifiées de disparité dans les sentences, on peut vouloir transformer cela dans un des buts, à savoir celui de réduire la disparité. Ces cinq problèmes buts de la Commission sont donc les suivants:

1) le problème de la disparité des sentences;

2) le problème de l'usage abusif de l'incarcération;

3) le problème du manque de transparence et de contrôle relié à l'exercice du pouvoir discrétionnaire (lack of accountability);

4) le problème de la durée imprévisible des peines d'incarcération;

5) le problème du manque d'information statistique systématique sur les sentences.

Pour ce qui est de la disparité des sentences: il semble désormais incontestable, nous dit la Commission, - tout au moins aux yeux de beaucoup de monde - qu'il existe des écarts injustifiés entre les sentences au Canada. La question de la disparité émerge donc avec d'autant plus de force qu'elle semble être à l'origine même de l'idée de constituer une commission pour se pencher sur les problèmes des sentences. En tout état de cause, l'importance accordée à cette question ressort de façon particulière de son mandat (p. 7-8). Le but de la Commission sera donc ici celui de réduire les disparités injustifiées de sentences. La Commission traitera de cette question sous l'égide du principe d'uniformité d'approche (p. 184; 448). 
Pour ce qui est de l' usage abusif de l' incarcération : la Commission ouvre son rapport (C.C.D.P., 1987:5) en rappelant les critiques virulentes faites à l'incarcération depuis l'époque de la construction du premier pénitencier canadien à Kingston. Elle souligne que l'on présentait déjà alors les prisons, entre autres, comme de véritables «écoles du crime payées par les citoyens" (pp. 5 et 19, note 1). Elle reprend aussi un reproche réitéré dans nombre de rapports officiels et dans la littérature criminologique et juridique canadienne: «il y a trop de monde dans les prisons, et les peines sont trop longues». La Commission identifie donc son premier problème: les prisons ne répondent pas - et ce depuis plus d'un siècle et demi de critiques et d'efforts de réformes - à nos attentes, produisent des "coûts sociaux" très élevés, coûtent économiquement très cher et leur usage est abusif. Cette pratique va à l'encontre du principe qui veut que l'emprisonnement soit une mesure de dernier ressort (Rapport Fauteux, 1956: 20) et peut être envisagée comme une atteinte aux droits fondamentaux de la personne (Canada, 1982: /5; 1984: 46). D'où un des buts de la Commission: réduire l'usage de l'emprisonnement.

Je voudrais procéder dès maintenant à deux mises en garde majeures. D'abord, par "usage" ou "recours", je désigne ici la double question de la fréquence d'emploi de cette mesure et de la longueur des peines. Ensuite, il faut éviter le piège de ne retenir de l'expression «recours excessif" souvent employée dans la littérature que sa dimension la plus superficielle. Elle renvoie, en réalité, à deux ordres d'idées. Parfois elle s'appuie sur une évaluation chiffrée portant sur le taux d'incarcération au Canada ou le nombre d'admission par jour. On dira alors, par exemple, qu'environ 30000 personnes sont incarcérées chaque jour et que notre taux d'incarcération est de 108 par 100000 habitants. Cette évaluation peut aussi comprendre une dimension comparative. On remarquera alors que ce taux est un des plus élevés du monde occidental (C.C.D.P., $1987: 255$ ). Mais, bien entendu, il ne faut pas accorder plus d'importance que nécessaire à ces arguments d' "impacts" ou de surface. Le deuxième sens du terme "excessif» est beaucoup plus important, car il touche davantage au fond du problème. "Excessif" veut dire dans ce cas plutôt «abusif», «inapproprié», «injuste». En ce sens, on peut parler de recours excessif à l'emprisonnement lorsque l'on ne choisit pas la mesure la moins restrictive qui soit suffisante et adéquate vu les circonstances (Canada, 1984:42; C.C.D.P., 1987:169). Un des termes importants de l'évaluation du "recours excessif" à l'emprisonnement relève donc de la recherche permanente d'un plus haut degré de respect des droits et libertés de la personne. 
Dans les termes de la Commission, le problème du recours abusif à l'emprisonnement fait partie d'une problématique plus complexe qui comprend deux autres problèmes: i) celui de l'éventail restreint de mesures à la portée du juge pénal et ii) celui de la difficulté concrète de tailler une place effective et importante pour ces mesures à l'intérieur de nos pratiques pénales actuelles. Ces deux facettes du problème sont reliées, mais ne se confondent pas. Car on pourrait disposer des mesures (dans le Code criminel, par exemple) sans réussir à les «introduire" pour autant de manière significative dans la pratique des tribunaux pénaux. Il faut donc à la fois proposer des «nouvelles» mesures et lever les obstacles éventuels à leur mise en pratique. La Commission traitera de l'ensemble de cette problématique sous l'égide du principe de modération'.

Pour ce qui est de la transparence du pouvoir discrétionnaire: ce problème-but de la Commission relève du caractère secret - et éventuellement dérogatoire et déconcertant - qui entoure certaines décisions importantes au niveau de la justice pénale (comme celles relatives aux pratiques d'inculpation de la police, jusqu'à la négociation du plaidoyer, à la justification des sanctions et aux décisions de la Commission de libérations conditionnelles, etc.). Globalement parlant, son but ici sera de trouver une procédure pour maximiser l'obligation du personnel de Justice de rendre compte de ses décisions. Ces questions seront notamment traitées sous l'égide du principe de la transparence du pouvoir discrétionnaire (p. 451).

Pour ce qui est, en quatrième lieu, de la durée imprévisible des peines d'incarcération: la Commission considère que les lois et pratiques actuelles en matière de détermination de la durée effective des peines d'incarcération sont à la fois ambiguës et confuses (p. 256-257). D'autre part, elle accorde beaucoup d'importance à la «signification apparente» des peines d'emprisonnement et semble soutenir que la décision initiale du juge concernant la durée d'une peine ne doit pas être revue plus tard par d'autres instances sur la base d'autres critères. Ce qui était alors considéré jusqu'aux années 70 comme une "vertu» du processus sentenciel («sa souplesse», sa «révision permanente» par différentes instances, etc.) devient en soi-même un problème. Son but ici sera d'éliminer

1. Nous avons analysé ailleurs l'évolution de ce principe dans les documents officiels canadiens (Pires, 1985) et la Commission fait part de certains de nos propos (p. 48-50). Ce qu'il faut souligner ici est que ce principe possède présentement différentes dimensions telles que, par exemple, celles de modération dans le recours au droit pénal, aux tribunaux, aux mesures plus punitives des tribunaux, à l'incarcération, dans la longueur des sentences d'incarcération, etc. La Commission fera surtout état de ces trois dernières dimensions. Le principe de la modération sera donc étroitement relié à celui de la sentence appropriée la moins lourde. 
l'imprévisibilité (plutôt que seulement l'ambiguité) dans le processus de la détermination de la longueur des peines. Cette question sera traitée sous l'égide du principe de la prévisibilité (en vue de favoriser l'imposition de sentences de durée réelle) (p. 271).

Pour ce qui est, enfin, du manque de renseignements sur les sentences : ce dernier problème est d'un statut difficile à préciser. Il prend tantôt un statut théorique, tantôt simplement pragmatique. Dans le premier cas, il prend l'allure d'une hypothèse explicative de la disparité des sentences: comme les magistrats ne peuvent pas se faire une idée globale des pratiques sentencielles, ils ne peuvent pas ipso facto s'ajuster par rapport à elles. Dans le deuxième cas, ce problème est vu comme un obstacle au bon fonctionnement de l'administration de la justice et à notre connaissance scientifique en vue de réformes (p. 65-68). Compte tenu du statut particulier de ce problème, il ne s'exprime pas - à proprement parler comme les autres en termes de "principes".

L'analyse du rapport que je me propose de faire ici va se concentrer, pour ainsi dire, sur le deuxième problème, c'est-à-dire la question de la modération dans le recours à l'emprisonnement et aux mesures communautaires. Certes, compte tenu du lien étroit entre les problèmes, je devrai parfois m'y référer, mais je ne le ferai que pour apporter quelques mises au point par rapport à mon thème. Soulignons, d'autre part, que même celui-ci ne pourra pas être traité (ou approfondi) dans toutes ses dimensions importantes. Avant d'entreprendre l'analyse proprement dite du rapport, je le situerai brièvement dans le cadre du débat pénologique contemporain.

\subsection{LA MATRICE GLOBALE DU RAPPORT: LE «MODĖLE (CLASSIQUE) DE JUSTICE»}

La littérature anglo-saxonne oppose deux grandes conceptions du droit pénal et de la justice, en l'occurrence le Justice Model vs le Medical Model (ou le Individualized Treatment Model) ${ }^{2}$. Cependant, il est important de souligner dès maintenant que depuis l'émergence du Medical Model au XIX ${ }^{e}$ siècle, aucun de ces deuX modèles n'a été "mis en pratique» de manière absolue. Cela veut dire qu'en pratique, le système

2. Voir, à ce propos, le rapport classique présenté par l'American Friends Service Committee (1971). Dans la littérature juridique de l'Europe continentale, cette polarisation des modèles trouve, dans une certaine mesure, sa contrepartie dans l'opposition que l'on fait entre le «droit pénal classique" et le «droit pénal d'inspiration positiviste». Sur cette opposition, et sur le compromis pratique qui en a découlé, voir l'intéressante étude de Tulkens et Digneffe (1981). 
pénal a toujours fait une sorte de compromis entre ces deux modèles, oscillant tantôt davantage dans une direction, tantôt davantage dans l'autre. Comme soulignent Tulkens et Digneffe (1981:201), les querelles d'École deviennent ici des "amalgames législatifs» et aussi, ajoutons-le, des amalgames philosophiques et théoriques au plan de la doctrine juridique et de la légitimation du droit pénal. Ceci dit, et afin de mieux visualiser la différence entre ces deux modèles, je les présenterai ici dans leur version théorique la plus orthodoxe. Car ils déterminent encore les grandes lignes de l'enjeu théorique et philosophique de la pénologie contemporaine.

Le Medical Model assume que le "crime" est le symptôme d'une pathologie individuelle et que le système pénal doit être conçu comme une forme de «traitement» et d'intervention préventive axée sur la prédiction de cette "maladie" chez l'individu. La philosophie pénale privilégiée ici est l'utilitarisme pénal axé soit sur la réhabilitation, soit sur la neutralisation, soit sur les deux à la fois. On revendique alors une très grande marge de pouvoir discrétionnaire afin de mieux adapter le «traitement" à l'infracteur et à l'évolution de la maladie chez lui sans égard à l'infraction comme telle (car elle n'est que le symptôme d'un problème dont la portée varie en fonction de l'accusé et non de la gravité de l'acte). La "punition» ou, si l'on veut, le «traitement» est présenté comme étant "pour le propre bien" du contrevenant (I'm punishing you for your own good) et de la société dans son ensemble. La peine la plus adaptée logiquement à ce modèle est sans aucun doute la sentence indéterminée. À tout le moins, faut-il s'assurer de pouvoir réagir énergiquement face à des "actes bénins" qui puissent signifier, aux yeux des évaluateurs, une "maladie grave». Bref, théoriquement parlant, ce modèle ne pose pas des limites a priori au pouvoir de punir. C'est ce modèle - semble-t-il, que l'on considère comme ayant prédominé (tout au moins au niveau du discours) depuis les réformes pénales de la fin du XIXe et du début du XXe siècles menées au nom de la "réhabilitation" (American Friends Service Committee, 1971 : 37). Quoi qu'il en soit, ce modèle a connu aux ÉtatsUnis, entre autres choses avec l'adoption des sentences indéterminées, un développement marqué jusqu' aux années 60 et est tombé en désuétude vers la fin des années 70 avec la crise de l'idéal de réhabilitation.

Le modèle (classique) de justice, par contre, assume que le contrevenant est un individu normal et rationnel qui a choisi librement de commettre une infraction. Il met l'accent sur la théorie du libre arbitre et sur les notions de culpabilité et de responsabilité pénales, aussi bien que sur celle de punition. Il peut abriter une ou plusieurs des philosophies pénales suivantes: le rétributivisme, l'utilitarisme pénal classique axé sur 
la dissuasion, la théorie du juste dû (Just Desert) et/ou celle de la dénonciation symbolique du comportement. Il faut remarquer que certains représentants contemporains de ce modèle soutiennent aussi l'utilitarisme pénal positiviste axé sur la neutralisation (qui est, soit dit en passant, le volet le plus conservateur du Medical Model). Les idées dominantes dans ce groupe, préconisées à la fois par les théories rétributivistes de la dissuasion et du juste dû, est qu' «il faut infliger au coupable le mal qu'il mérite» et que les peines doivent être déterminées et prévisibles. Ce modèle assume en outre que la justice pénale est et doit être guidée par les principes abstraits du droit et de la procédure pénale (le due process of law), et se méfie du pouvoir discrétionnaire des bureaucraties et du manque de transparence (lack of accountability) des décisions. Il valorise donc la "certitude» et la «clarté», voire la publicité. En règle générale, le modèle insiste sur la nécessité de limiter a priori le pouvoir de l'État de punir par l'entremise, entre autres, du principe de proportionnalité de la peine. La théorie contemporaine du juste dû, quant à elle, prône explicitement le principe de modération dans l'usage de l'emprisonnement.

Dans cette conception classique du droit pénal, on suppose (explicitement ou implicitement) que la sanction pénale possède quelques attributs spécifiques qui la distingueraient fondamentalement de la sanction civile. On suppose d'abord que seule la sanction pénale punit; on suppose ensuite qu'elle est plus efficace pour régler certains problèmes justement parce qu'elle porterait en elle-même un plus grand potentiel dissuasif que la sanction civile. Il s'ensuit de là que le rôle du juge pénal (au niveau de la sentence) est conçu, règle générale, tout simplement comme celui de punir (équitablement) l'inculpé (pour protéger le public, pour dissuader, etc.).

Cela signifie que, dans ce modèle, tant en théorie qu'en pratique, il n'y a pas de place importante pour les mesures réparatrices, pour la médiation et/ou la réconciliation, pour les avertissements, pour l'ordonnance d'engagement, etc. ${ }^{3}$. Car ces mesures sont identifiées au droit civil et conçues simultanément comme n'ayant pas de contenu punitif, ne remplissant pas les finalités du droit pénal et ne relevant pas du rôle du juge pénal. On privilégiera alors plutôt les peines classiques, comme l'amende, l'emprisonnement et le sursis (parfois la probation).

3. Ce genre de raisonnement est présent même chez un des grands représentants de la théorie du juste dû qui préconise pourtant la modération dans le recours à l'emprisonnement (voir von Hirsch, 1986, particulièrement le chap. 5). C'est que modération et innovation en termes pénologiques ne relèvent pas toujours d'une même démarche intellectuelle ou cheminement théorique. 
La Commission s'approche plutôt de ce modèle classique de justice, tout en essayant de s'en démarquer sur des points spécifiques. C'est dans la conception de la peine, de la punition et du rôle du juge pénal qu'elle s'éloignera le plus de ce modèle. Elle prendra alors une position diamétralement opposée en ce qui concerne les mesures réparatrices et communautaires. Par contre, c'est dans la façon de concevoir la "prévisibilité» par rapport à la durée des peines et les moyens pour «rendre transparentes» les décisions que la Commission s'approche le plus du Justice Model. C'est par l'entremise d'une forte concentration du pouvoir au niveau de la cour (au détriment des autres instances et acteurs du système) qu'elle entreprendra ses recommandations en vue de «rendre transparentes» les décisions.

Je pense qu'on peut dire sans risque d'erreur que la Commission élabore son orientation spécifique et originale à partir de la matrice fournie par le Justice Model . Nous définirons ici sa position comme étant celle d'un classicisme pénal modifié ou, mieux encore, éclaté.

\subsection{LA DÉCLARATION DU BUT ET DES PRINCIPES DE LA} SENTENCE: DES THÉORIES DE LA PEINE À UNE THÉORIE DE LA SANCTION

La Commission se propose d'articuler une (nouvelle) "théorie" du but et des principes de la sentence pour la substituer aux «théories (du but) de la peine" (rétributivistes, de la dissuasion, de la réhabilitation, de la neutralisation, du juste dû et/ou de la dénonciation) qui sont présentement mises à contribution par la cour au moment de la détermination de la sanction (p. 118). On peut dégager à cet égard plusieurs raisons (explicites ou implicites dans le rapport) qui militent en faveur d'une telle décision.

\subsection{DE L'INOPPORTUNITÉ DES «THÉORIES (BUTS) DE LA PEINE»}

D'abord, les «théories de la peine» sont considérées à l'heure actuelle, tant par la Commission (p.58-60) que par une bonne partie de la littérature canadienne (Pires, 1984), comme une source éventuelle de disparité au niveau des sentences. Comme les magistrats se différencient, entre autres choses, par leur philosophie pénale (leur «théorie de la peine») et adoptent des principes sentenciels complètement différents, voire opposés, on constate des divergences marquées sur presque tous les aspects de la détermination des sentences (Decore, 1964; Hogarth, 1971, etc.). D'autre part, et cela indépendamment des autres problèmes, il semble difficile de vouloir prendre parti pour une seule de ces "théories" 
et invraisemblablement de vouloir l'imposer aux juges dans tous les cas qui se présentent devant eux. Aucune de ces théories de la peine (la dissuasion, la réhabilitation, etc.) ne sont inconditionnellement reçues ou recevables à titre de "but général» de la sentence par les tribunaux. Qui plus est, on constate parfois dans une même décision un mélange de théories de la peine (qui sont vraisemblablement contradictoires sur le plan théorique) sans pouvoir comprendre exactement de quelle façon elles ont affecté la décision en cause. Ce problème, qui remonte au XIX ${ }^{e}$ siècle, a des implications pratiques particulièrement importantes dans les pays où - comme chez nous - le Code criminel accorde, comparativement à d'autres codes de la tradition romaniste, un énorme pouvoir discrétionnaire aux juges en ce qui concerne le choix du type de sanction et le quantum éventuel de celle-là.

Deuxièmement, les théories de la peine posent par elles-mêmes plusieurs problèmes concernant à la fois leur propre validité et leur portée théorique et pratique. Je renvoie ici le lecteur aux critiques internes de la Commission sur ces théories à partir, entre autres choses, des résultats de recherches (p. 147-159). Il suffit de rappeler que ces problèmes mettent encore une fois en doute toute prévention à accorder à une quelconque de ces théories (dans leur version courante) une place centrale au niveau de la détermination de la sentence. Ceci signifie concrètement que, pour la Commission, le «but» du juge au niveau de la détermination de la sentence n'est donc ni spécifiquement ni fondamentalement celui de «rétribuer le mal par le mal au nom d'une quelconque exigence de justice (ou de droit), ou celui de "réadapter le contrevenant», ou celui de "le punir" ou celui de "séparer les contrevenants jugés dangereux de la communauté" ou encore celui de «dissuader les contrevenants potentiels» de poser des actes susceptibles de tomber sur le coup de la loi pénale.

Troisièmement, la Commission ne croit pas, à juste titre, que le "but" général de "protection de la société» (ou du "public») — que l'on attribue parfois également à l'ensemble du droit pénal - puisse être appliqué sans soulever des problèmes majeurs et sans créer des risques importants (relatifs à un usage abusif de l'emprisonnement) au niveau de la détermination de la sanction (p. 160-164)4 ${ }^{4}$ En effet, le but de pro-

4. La protection de la société n'est pas à proprement parler une «théorie de la peine», mais plutôt une finalité attribuée par certains juristes et philosophes du droit à l'ensemble du droit pénal. Cependant, on trouve dans la jurisprudence canadienne et dans quelques études juridiques ou criminologiques une transposition automatique (et indue) de cette "finalité" du droit pénal au moment de la détermination de la sentence. C'est pour cette raison que la Commission attire l'attention ici, à son tour, sur le caractère inapproprié d'une telle démarche. 
tection de la société (ou du public) est à la fois trop général, irréaliste et susceptible d'être associé indûment à la prison; trop général, d'abord, car plusieurs ministères (Agriculture, Environnement, de la Santé et du Bien-être social, etc.) et organismes gouvernementaux (la police, l'armée, les pompiers, etc.) pourraient prétendre aussi à bon droit que c'est également leur objectif global (p. 163); irréaliste, ensuite, car il risque de créer des fausses attentes et de graves malentendus relatifs aux exigences de justice et d'équité qui reviennent en première ligne aux tribunaux (p. 163-164); susceptible finalement d'être associé indûment à la prison, car certaines études sur la sentence, la jurisprudence et la pratique judiciaire ont souvent assimilé la "protection du public" à la neutralisation par l'incarcération (p. 161; Canada, 1984: 38). Bien que le terme "protection" soit aussi employé couramment dans le domaine des assurances pour désigner le recouvrement (la «compensation») des pertes, en droit pénal, ce terme a la fâcheuse tendance à exclure les mesures réparatrices. On oublie qu'il y a différentes formes de protection et que le dédommagement en est une. D'autre part, les objectifs de "justice" (p. 163) et de recherche de la mesure appropriée la moins lourde — qui reviennent plus directement aux tribunaux - sont plus complexes que le simple recours à l'emprisonnement implicite dans une certaine interprétation de l'expression "protection de la société». Voilà quelques-unes des raisons qui amènent la Commission à écarter une simple transposition de cette «théorie du droit pénal" au plan de la sentence.

Finalement, ces «théories de la peine» présentent deux autres inconvénients majeurs qui sont d'ailleurs étroitement reliés, même s'ils ne sont pas tout à fait identiques. Le premier inconvénient est posé explicitement par la Commission. On peut le résumer ainsi: les théories existantes de la peine conçoivent en quelque sorte l'activité (et le rôle) du juge au niveau de la sentence comme étant fondamentalement d'ordre punitif. Elles mettent toutes l'accent sur la punition et la peine au sens fort et classique de ces termes (voir p. 115-119). D'où le deuxième inconvénient qui est un corollaire du précédent et n'est posé qu'implicitement par la Commission: les théories («buts») de la peine ne servent pas de support (théorique) aux sanctions communautaires (notamment celles de nature réparatrice ou relevant de la médiation) et/ou n'ont pas été conçues en fonction d'un éventail plus vaste de sanctions. Au contraire, certaines de ces théories (les rétributivistes, de la dissuasion, du juste dû et de la neutralisation) non seulement font silence sur ces mesures réparatrices mais comme souvent ferment les portes à ces mesures en évoquant tantôt une éthique rigoriste de la peine qui a pour effet de transformer la "punition classique» en une sorte d'impératif moral, tantôt une utilité 
sociale (e.g., la dissuasion) qui serait l'apanage exclusif de la peine, tantôt encore les deux à la fois. Or, la Commission veut justement promouvoir l'adoption de nouvelles sanctions réparatrices «autonomes" (à l'instar du dédommagement) au bénéfice des victimes et/ou un usage accru des sanctions communautaires existantes. Il faut donc envisager différemment le but des sanctions en droit pénal et concevoir autrement le rôle du juge au niveau sentenciel.

Il faut remarquer ici que même la "théorie du juste dû" — qui exerce à maints égards une attraction particulière sur la Commission - constitue pour elle, sur le plan spécifique des mesures réparatrices, plutôt un obstacle de taille qu'une bouée de sauvetage. En effet, la théorie du juste dû a tendance à mettre exclusivement l'accent sur la punition, et ce malgré sa prise de position en faveur de la modération. C'est que le principe de la proportionnalité - selon lequel la punition doit observer les proportions de la gravité de l'infraction - et le principe (partiellement implicite) de l'obligation de punir de manière semblable tous les cas qui ont fait formellement l'objet d'une même condamnation pénale deviennent ici la clef de voûte de cette théorie. Par conséquent, elle laisse dans l'ombre - voire ferme les portes à - la question de la recherche de la mesure appropriée eu égard aux circonstances. Pour cette théorie, modération veut dire alors simplement "punir parcimonieusement"; modération dans la punition. À la question «pourquoi doit-on punir parcimonieusement?» la théorie ne répond grosso modo que ceci: parce que nous savons que ce n'est pas la sévérité des peines qui compte le plus et que punir trop provoque des effets négatifs divers.

D'où le fait que la modération prend effectivement, dans la théorie du juste dû, l'allure d'un simple appel à l'indulgence ou à la sagesse 5 . D'où aussi le fait que cette théorie, même lorsqu'elle stimule le recours aux sentences non carcérales, ne réclame pas l'élargissement des mesures sentencielles ni une plus grande place pour les mesures réparatrices tout court dans le cadre des solutions pénales. Qui plus est, tantôt elle fait silence sur la médiation et/ou la réconciliation, tantôt elle écarte ces mesures comme non pertinentes. Elle se renferme ainsi dans le cadre pénologique classique: sursis, amende, probation, courtes peines d'emprisonnement.

La Commission semble donc éprouver le besoin de se débarrasser du carcan imposé par les théories de la peine. En effet, à défaut de ne pas

5. Si l'on voulait résumer brutalement la théorie du juste dû, on dirait ceci : c'est la théorie selon laquelle il faut punir proportionnellement, punir équitablement, punir parcimonieusement, mais surtout toujours punir. 
pouvoir trancher d'un seul coup, il faut défaire patiemment le "nœud gordien» qui attache la pratique sentencielle à ces théories. C'est, entre autres, ce que vise la Commission en proposant une «nouvelle théorie de la sentence» dans sa Déclaration du but et des principes de la sentence.

La Commission a fondamentalement raison à propos des deux derniers inconvénients indiqués ci-dessus, mais une dimension du problème relative à la "théorie de la réhabilitation» lui a échappé. Je présenterai d'abord la "théorie de la sentence» de la Commission qui constitue sa réponse au problème posé par les théories de la peine. Ensuite, je ferai quelques remarques critiques sur sa façon d'envisager la théorie de la réhabilitation (en particulier par rapport aux mesures communautaires).

\subsection{LA DISTINCTION FONDAMENTALE ENTRE LA SENTENCE ET LA PEINE}

Afin d'écarter cette assimilation à la fois abusive et encombrante entre les théories de la peine et la pratique sentencielle, la Commission cherche alors à distinguer sentence (ou sanction) de peine, de punition. En effet, elle soutient "qu'il y a lieu de remettre en cause l'identification actuelle de la sentence à la punition" (p. 115). On ne doit donc pas confondre "théorie de la sentence» avec «théorie de la peine", car la seconde est beaucoup plus restrictive que la première. D'autre part, ces théories ont une tendance soit à travestir la dimension punitive de la pratique sentencielle actuelle en la présentant comme un simple traitement "pour le propre bien du contrevenant", soit encore à favoriser une attitude répressive qui consiste à affirmer que le «but» des juges pénaux est celui d' «infliger au coupable le mal qu'il mérite» (p. 118).

Qu'est-ce que veut dire alors une (nouvelle) théorie de la sentence? Et comment en sommes-nous venus à identifier sentence et peine? Est-ce que ces termes veulent vraiment dire la même chose?

Aujourd'hui, dans le droit pénal canadien (qui suit à cet égard la tradition du common law), ces termes sont devenus à toutes fins pratiques synonymes. Il y a seulement une «zone nébuleuse» qui concerne le statut juridique de la libération inconditionnelle. Elle est sans aucun doute une "sentence», mais on est généralement d'accord pour dire que le terme "peine» s'applique mal ici (p. 120). En dehors de ce cas, les deux termes sont largement interchangeables. Les deux phrases suivantes sont alors équivalentes: «le juge a imposé une peine à l'accusé» ou encore «le juge a imposé une sentence à l'accusé". 
À l'origine cependant, le terme «sentence» n'avait pas ce sens (de "punition") qu'on lui donne aujourd'hui chez nous. En effet, le mot sentence vient du verbe "sentir" (du lat. sentire, senus) qui signifie "éprouver un sentiment", d'où sentence (du lat. sententia): «impression de l'esprit", «avis», "décision». La sentence était étymologiquement une décision, un jugement (et non une peine). D'ailleurs, dans plusieurs pays où le droit a suivi la tradition romaniste, ce terme continue à garder son acception d'origine. On parlera alors, à l'instar du droit de procédure pénale brésilien aussi bien de «sentence condamnatoire» (Code de proc. pénale, art. 385) que de «sentence d'acquittement» (art. 386). En outre, le terme sentence est employé autant en droit pénal qu'en droit civil. On parlera alors de "sentence de séparation des époux" («sentença de desquite», art. 322 du Code civil), etc.

Notre droit canadien, par contre, emploie ce terme «sentence" seulement en matière pénale. En outre, il distingue clairement entre le moment de la culpabilité (le verdict de culpabilité ou d'acquittement) et celui de la peine (Béliveau et Pradel, 1986: 249). Et il a réservé le terme "sentence» pour désigner ce dernier moment. Cela veut dire que le prononcé de la «sentence» vient, pour ainsi dire, "après» (i.e., n'inclut pas) le verdict de culpabilité, et ce, même si elle est prononcée immédiatement après (i.e., sans recours à une "audition présentencielle»). D'où, probablement, une des raisons qui peuvent expliquer l'identification pratique des deux termes.

Quoi qu'il en soit, il me semble que si la Commission cherche à dissocier la conception de la sentence de celle de la peine, c'est moins en raison de la perte du sens du terme "sentence" que des problèmes entourant le sens actuel du terme "peine» 6 . Son but est de substituer la notion plus large de sanction légale à la notion plus stricte de peine. Le rôle du juge demeure (parmi d' autres) celui de donner une "sentence" (au sens du droit canadien), mais celle-ci ne doit pas être conçue au sens strict de "peine», "punition", mais plutôt au sens large de "sanction légale».

6. Je parle ici de «sens actuel» parce que les termes «peine», "pénai», si l'on remonte à leur origine, viennent du grec poinê qui signifie «prix du sang", "l'argent qu'on paie aux parents de la victime d'un meurtre*. Ils nous sont arrivés à travers le latin apoena» qui signifiait aussi d'abord «rançon d'un meurtre», «réparation», puis punition au sens que nous lui donnons aujourd'hui. Ces termes avaient donc au début de toute évidence le sens d'une "réparation pécuniaire" (au plus, une punition réparatrice) qui s'adressait directement aux parties impliquées (à la victime ou sa famille), avant d'assumer le sens plus tardif où la dimension de «punition symbolique» et de toute espèce de châtiments sur la personne l'emporte sur celle de "dédommagement" ou de «punition réparatrice* et où l'État non seulement prend la place de la victime en cas de réparation pécuniaire (l'amende), mais prime sur elle sur tous les aspects de la procédure judiciaine. L'État n'est pas un éventuel médiateur-modérateur, mais plutốt la Victime, la "Victime au nom de tous". 
En effet, pour la Commission, la «sentence est la détermination judiciaire d'une sanction légale devant être imposée à une personne trouvée coupable d'une infraction» (p. 126). Sentence devient alors plutôt synonyme de sanction que de peine.

Bref, pour la Commission, la notion de peine et de punition dans son acception stricte (et/ou classique) est incompatible avec son objectif de modération et de mieux outiller le juge pénal. D'abord, elle nous renvoie à un éventail trop réduit de sanctions possibles où l'emprisonnement prend l'allure de la "sanction normale" à être imposée, les autres ne constituant que des simples «alternatives" (p. 85-86). Ensuite, elle exclut le dédommagement et la restitution (surtout s'ils ne sont pas accompagnés de dommages-intérêts) à titre de sanctions principales et suffisantes en soi-même. Ils sont alors rélégués au statut de «mesures civiles complémentaires», de simples «circonstances atténuantes», etc. Finalement, cette notion caractérise mal la mesure de libération inconditionnelle.

En outre, la Commission signale que la notion de «sentence" (en droit canadien) englobe deux idées distinctes: celle d'obligation (ou de coercition) et celle de punition (stricto sensus) (p. 126).

Toute sentence porte en elle la notion d'obligation, car elle doit être nécessairement exécutée. Certes, la Commission laisse entendre qu'il y a deux formes principales d'obligation. Dans la première, l'obligation dans l'exécution repose essentiellement et en première instance sur l'inculpé (par exemple, le paiement d'une amende). Dans la deuxième forme, l'obligation dans l'exécution ne relève plus de la même façon de l'inculpé. Au contraire, la part de l'État (ou des autorités correctionnelles) passe également au premier rang et augmente considérablement selon les sanctions jusqu'à avoir la priorité. Tel est le cas de la probation et, à plus forte raison, des (longues) sentences d'incarcération. Il n'en demeure pas moins que l'idée générale d'obligation est toujours fortement présente dans toutes les sanctions.

Qu'en est-il de l'idée de punition? Sous un certain angle, dira la Commission, elle est aussi présente partout en ce sens que toute sanction a des conséquences négatives à des degrés divers. Qui plus est, faire l'objet de coercition est toujours déplaisant. Mais cette acception large de punition a l'inconvénient de se confondre avec la notion d'obligation et perd toute sa spécificité. La Commission propose alors de distinguer la notion d'obligation de celle de punition en fonction du degré de privation qu'elles imposent à l'inculpé. Dans ce sens, «toutes les sentences n'imposent pas un degré de privation tel qu'on puisse légitimement les appeler punitions» (p. 126). Elle en donne deux exemples clairs: la libération 
inconditionnelle et le dédommagement sans dommages exemplaires. Il est toujours déplaisant - et parfois voire très pénible - d'avoir à dédommager, mais le degré de privation ici tout au moins ne «déborde» pas les conséquences de la conduite.

La notion de punition (stricto sensu) est donc beaucoup plus restrictive que celle d'obligation et, pour cette raison, elle ne sert pas à caractériser l'ensemble des sanctions, et moins encore le rôle du juge dans le cadre des nouveaux paramètres proposés par la Commission. Celle-ci souligne alors que "la notion d'obligation prime sur celle de punition" (p. 126) en ce sens que "la notion d'obligation comprend celle de punition, alors que la réciproque n'est pas vraie" (p. 126). J'attire ici l'attention sur l'ambiguité de ce passage dans la traduction française du rapport. Comme nous l'avons vu ci-dessus, la Commission ne veut pas dire que l'idée de punition (stricto sensu) exclut celle d'obligation (ce qui serait d'ailleurs absurde), mais tout simplement que celle-ci a une acception plus large que celle-là ("the notion of obligation is more comprehensive than the notion of punishment , p. 115).

Mais la notion d'obligation prime aussi sur celle de punition en cet autre sens plus fondamental que, pour la Commission, il est plus important d'obliger queiqu'un à rendre compte de ses actes à la Justice que de punir en tant que tel. Elle dira alors dans sa Déclaration du but de la sentence que «l'affirmation de la responsabilité sociale du contrevenant (the accountability of the offender) plutôt que sa punition (est) le facteur primordial" (p. 169). Cela signifie concrètement que l'on peut affirmer la responsabilité sociale d'un individu sans recourir à la punition (stricto sensu).

La Commission introduit par la suite une distinction complémentaire dont l'importance pour la compréhension de l'ensemble de son modèle est capitale: celle entre les sanctions carcérales et les sanctions communautaires (p. $128 ; 381$ et suiv.). Soulignons dès maintenant que l'expression "sanctions communautaires" - jusqu'ici très fortement associées aux ordonnances de travaux communautaires - s'applique à toutes les sanctions (amende, probation, etc.) qui ne constituent pas une forme quelconque d'emprisonnement ${ }^{\text {? }}$.

7. L'assignation à domicile sous surveilłance électronique, comme le souligne Landreville (1987), est une forme d'emprisonnement. Si cette mesure devient acceptée chez nous, elle doit donc être considérée comme une «sanction carcérale» ou, plus précisément, d'emprisonnement et jamais comme une sanction communautaire. C'est de la pure publicité commerciale que de la présenter comme une "alternative à l'emprisonnement». La Commission semble, hélas, classer présentement ce type de mesure parmi les sanctions communautaires (p. 129). Comme nous le verrons, cette option, qui relève sans aucun doute d'un 
Elle opère cette distinction - que je trouve fort intéressante - à partir des critères reflétant «la nature des sanctions en cause» (p. 381). J'ai dégagé trois critères dont deux sont implicites dans le rapport.

Le premier critère - dont la portée opérationnelle est la plus évidente - se réfere, pour ainsi dire, au lieu d'exécution de la sentence: au sein de la communauté ou, à contrario sensu, en ostracisme, en ségrégation (p. 128). Ce critère n'est cependant pas purement opérationnel, parce qu'il porte en lui des significations particulières qu'il convient de prendre au sérieux. Si le juge choisit une sanction communautaire, c'est parce qu'il ne considère ni nécessaire ni appropriée une sanction de ségrégation. Il faut donc éviter de passer trop vite de la sanction communautaire à la sanction d'incarcération comme il arrive présentement avec le non-paiement d'une amende (p. 128)

La distinction précédente entre la notion d'obligation et celle de punition ne recoupe pas entièrement celle entre ces deux grands types de sanctions, mais aide à mieux les saisir. En effet, une sanction communautaire peut avoir parfois une dimension fortement punitive (au sens strict). Par exemple, un dédommagement avec des dommages exemplaires très élevés (par rapport au revenu de l'inculpé) ou encore une amende sévère. Par ailleurs, ces sanctions, par la forme d'obligation qu'elles assument, ont tendance à affirmer (positivement) la responsabilité sociale du contrevenant. Au contraire, une sanction carcérale sera toujours punitive, une "peine», au sens propre du terme. D'autre part, la forme d'obligation change complètement et sans ambiguité chez les sanctions carcérales: l'individu est ici "entièrement pris en charge", investi dans la totalité de sa personne par l'État; il devient, à l'égard de l'exécution de sa sentence, particulièrement "passif" et «dépendant"; et ce à tel point que l'on peut dire qu'il «subit» plutôt qu'il n'assume sa responsabilité sociale.

Si nous approfondissons librement ces critères de la Commission, nous pouvons donc dire que la "forme globale de la réaction" change

cas limite, me paraît cependant plutôt en contradiction avec les fondements mêmes de cette distinction proposée par la Commission. Par ailleurs, elle semble s'opposer clairement à l'adoption de cette mesure (p. 403). En ce sens elle fait écho au rapport du Comité d'étude sur les solutions de rechange à l'incarcération du Québec (1986).

8. Cet argument s'applique, mutatis mutandis, à l'assignation à la maison sous surveillance électronique. Le lieu d'exécution perd ici sa véritable signification théorique et pratique, car la nature de la sanction change radicalement. L'accusé est ici en état de ségrégation, ce qui change est le degré de la ségrégation (ce degré change aussi d'une prison à sécurité maximale à une prison à sécurité minimale ou encore à l'emprisonnement en milieu ouvert). En outre, il sera plus facile de contrôler l'usage abusif de cette mesure (dans les lignes directrices) si on la considère comme une forme d'emprisonnement. 
également d'un type de sanction à l'autre. Ce changement constitue d'ailleurs la dimension qualitative du degré de privation que l'on impose au contrevenant. Parce que les sanctions carcérales accaparent l'individu tout entier, elles sont à cet égard beaucoup plus «totales», dégradantes et aliénantes; elles impliquent aussi une plus grande part d'abus de pouvoir et, comme remarque Goffman (1968: 95), ressemblent davantage au système de punitions réservées en général aux animaux et aux enfants (avec la différence importante qu'elle ne se situe pas, comme généralement dans le cas des enfants, dans le cadre d'une relation affective). Si j'emprunte ici la terminologie de Goffman (1968: 41), je dirais que les sanctions carcérales sont des "sanctions totalitaires" au double sens du mot : elles portent sur l'individu tout entier (aussi bien que sur différentes dimensions de sa vie) et elles subissent en permanence un forte «tentation totalitaire" au sens moderne du concept.

Le schéma ci-dessous résume les positions de la Commission telles que développées ici:

1) Sanctions communautaires

a) lieux d'exécution: au sein de la Communauté;

b) idée force: obligation, coercition, affirmation (positive) de la responsabilité;

c) forme de réaction/punition: spécifique; non ou moins aliénante (en soi-même).

2) Sanctions carcérales

a) lieu d'exécution: en ségrégation; séparé de la communauté;

b) idée force: punition;

c) forme de réaction/punition: désintégrative (en soi-même); «totalitaire».

La distinction entre "peine» et "sanction légale" étant faite, quel sera alors le (nouveau) but de la sentence (ou des sanctions légales) et quels seront les nouveaux principes régissant sa mise en pratique? Voilà ce dont je traiterai ci-dessous.

\subsection{LA «RESPONSABILITÉ SOCIALE» DES CONTREVENANTS ET LA RECHERCHE DES «SANCTIONS JUSTES»}

Pour la Commission, l'idée centrale dans la détermination de la sentence est celle de rendre compte de nos comportements à la Justice: «le but essentiel de la sentence consiste à préserver l'autorité de la loi et à 
en promouvoir le respect par l'imposition de sanctions justes». Préserver l'autorité de la loi signifie tenir les gens responsables au moyen des sanctions (et pas nécessairement des "peines»), «des actes qui trahissent les valeurs fondamentales de leur collectivité» (p. 165-166).

Le juge n'aura donc comme fonction essentielle au niveau de la pratique sentencielle ni celle de «réduire le taux de criminalité», ni de "punir", ni encore de "réadapter", ni de "séparer les contrevenants de la société», ni même de compenser le préjudice causé à la victime ou à la collectivité: c'est fondamentalement - le cas échéant - celle de tenir les gens responsables par l'imposition de sanctions justes. Selon la déclaration du but de la sentence, le juge peut s'arrêter à l'une ou plusieurs des considérations mentionnées ci-dessus, mais elles ne doivent plus guider fondamentalement ou exclusivement son choix de la sanction.

Quels sont alors les principes qui doivent (théoriquement) commander le choix des sanctions et donc déterminer le sens de l'expression «sanction juste»?

La Commission retient deux grands principes qui ne sont pas à proprement parler complémentaires. Au contraire, ils gardent une certaine tension entre eux : le principe de la proportionnalité et le principe de la sentence appropriée la moins lourde. Hélas, la Commission a accordé le rôle prépondérant dans la détermination d'une «sanction juste» au principe de la proportionnalité (p. 169). Pourtant c'est le deuxième principe qui exprime le mieux le principe de la modération. C'est que le principe de la proportionnalité conserve ici une flamme rétributiviste évidente. En effet, la proportionnalité est, dans le cadre du droit pénal, un principe à double tranchant. Lorsqu'on dit que «la punition doit observer les proportions de la gravité de l'infraction", on passe en réalité un double message: il faut, bien sûr, ne pas excéder la gravité de l'infraction ( le "nec plus ultra»), mais il faut aussi assurer que la punition soit infligée et éventuellement qu'elle ne soit pas non plus «moindre» que l'évaluation de la gravité de l'infraction faite par le juge. En outre, tel que formulé dans la Déclaration du but de la sentence, le principe de la proportionnalité constitue aussi une réserve au principe de la sentence appropriée la moins lourde. Il est donc là moins pour limiter le pouvoir de punir (ce que le deuxième principe fait déjà en mettant l'accent sur la sentence «la moins lourde") que pour permettre de rehausser la dimension punitive de la sanction au-delà de ce qui serait approprié eu égard aux circonstances.

Comme nous le verrons, les propositions de la Commission n'ont pas pris beaucoup en considération le principe de la modération et/ou de la sentence appropriée la moins lourde dans le cas des infractions graves 
contre la personne (et peut-être dans certains cas d'infractions contre la propriété). Il est probable que le principe de la proportionnalité soit désigné particulièrement pour ces cas. Or, comme Brodeur (1986:165169) nous le démontre, c'est justement à l'égard de ces infractions que ce principe est le plus vague, «brutalement déproblématisé» et destitué de tout contenu précis, une véritable notion piégée. C'est que la "proportionnalité", le nec plus ultra, lorsqu'on la traduit en termes de punition sur la personne (et particulièrement en matière d'infractions contre la personne), correspond justement à l'absolument indéterminé et indéterminable, à une «enveloppe vide». Car aucune sanction ne peut -- ici à plus forte raison qu'ailleurs - "restituer» ou «effacer" (voire "correspondre» à) ce qui a été fait. La «proportion» ici est donc tout simplement l'expression du néant. Elle cède alors la place à une revendication rétributiviste (subjective) impossible à délimiter objectivement. Si l'on met le choix et le calcul de la punition à la remorque de notre indignation, on risque de grossir celle-là au-delà de toute limite raisonnable. À mon avis, ce principe sert donc davantage pour justifier certaines pratiques punitives actuelles sur lesquelles on n'est pas prêt à revenir qu'à orienter réellement le choix d'une "sanction juste».

Ces remarques ne doivent pas cependant nous faire oublier que le principe de la sentence appropriée la moins lourde demeure le «facteur primordial" (Déclaration..., p. 169), et qu'il l'emporte sur toutes les théories rétributivistes et utilitaristes de la peine tout au moins par rapport à un grand nombre d'infractions contre la propriété.

D'autre part, en introduisant les soi-disant «sanctions civiles» dans l'outillage du juge pénal et en parlant désormais de sanctions légales plutôt qu'exclusivement des peines, la Commission met implicitement en doute l'existence d'attributs spécifiques propres aux «peines». En effet, elle souligne d'abord que les mesures réparatrices ont aussi une dimension punitive et/ou déplaisante qu'il ne convient pas de prendre à la légère (mềme si l'on ne l'appelle pas "punition" au sens strict du terme) (p. 126-129; 396-397). Ensuite, elle met indirectement en valeur le potentiel dissuasif de toutes les sanctions dans leur ensemble (et non seulement celui de la sanction pénale classique) dans la mesure où elle souscrit aux résultats des recherches qui indiquent que la certitude et la fréquence de l'intervention est plus importante que la sévérité de la sanction (p. 148-151).

Par rapport à la conception du rôle du juge (pénal), les changements ne sont pas moins importants: il doit mettre désormais l'accent sur le choix de la sentence appropriée la moins lourde eu égard aux circonstan- 
ces, reléguant au deuxième plan les considérations classiques qui avaient tendance à l'orienter vers les sanctions carcérales. Il est censé aussi accorder une attention particulière aux droits de la personne. À mon avis, cette nouvelle conception du rôle du juge réclame encore des développements plus poussés et plus explicités.

\subsection{LA COMMISSION FACE À LA THÉORIE DE LA RÉHABILITATION}

Il me paraît important de bien comprendre la portée et les limites de la critique que la Commission adresse à la théorie de la peine axée sur la "réhabilitation du contrevenant».

Remarquons d'abord que compte tenu des recommandations que la Commission fait dans le sens d'abolir la libération conditionnelle et de réduire la remise des peines, il ressort du rapport que c'est à cette théorie qu'elle s'attaque le plus. Ainsi, en parlant de la libération conditionnelle, la Commission dira qu'il s'agit d'un système qui «repose essentiellement sur une théorie qui n'est plus reconnue comme fondée» (p. 72).

Écartons d'emblée, cependant, une erreur possible d'interprétation: la Commission ne met pas en cause la théorie de la réhabilitation dans son ensemble. En effet, si l'on tient compte des autres remarques de la Commission - y compris le fait qu'elle n'exclut pas complètement le principe de réadaptation de sa déclaration du but de la sentence (au même titre que la dissuasion, etc.) (p. 170), qu'elle recommande au juge de considérer ce but dans le cadre de programmes non carcéraux (p. 152) et qu'elle ne recommande pas l'abolition de la probation (qui est fondée sur cette même théorie) - il faut interpréter sa position de la façon suivante: ce qui est non fondé ce n'est pas la théorie de la réadaptation dans son ensemble et dans toutes ses significations, mais plutôt la partie de cette théorie qui prétend que le pénitencier et la prison sont des lieux de réhabilitation (et éventuellement aussi celle qui prétend que le crime est le symptôme d'une anormalité individuelle).

Qui plus est, la Commission considère «qu'il serait rétrograde de supprimer les mesures les plus humanitaires qui sont à la disposition des détenus" (p. 272) et reconnaît l'importance de programmes d'aide facultatifs au moment de leur libération (p. 278-279).

En d'autres mots, il faut dissocier la réadaptation de l'emprisonnement (p. 152). Mais pourquoi faire? La position de la Commission est ambiguë. En effet, comme nous avons vu, la Commission met explicitement en cause l'attitude de condamner quelqu'un à une peine d'emprisonnement sous prétexte de le "réhabiliter». Mais cette critique, juste en 
soi-même, réclame moins la mise en cause de la théorie de la réhabilitation que la mise en cause de la pratique de l'incarcération. On peut même critiquer cette pratique au nom de l'idéal de réhabilitation. C'est d'ailleurs ce que l'on a fait historiquement.

Chez nous, cette critique du pénitencier au nom de la théorie de la réhabilitation trouve un point tournant dans le rapport Fauteux (Pires, 1985:171; C.C.D.P., p. 49). À partir de ce moment, le pénitencier perd de plus en plus la faveur de la théorie de la réadaptation. Celle-ci commence à privilégier clairement les "mesures communautaires» (Fauteux, 1956: 13,15-16,49) et à s'opposer aux peines d'incarcération trop longues parce qu'elles sont source de maints problèmes, dont la création de la dépendance chez la personne. Fauteux (1956:50) critique déjà les peines de 8, 10 ou 15 ans en les considérant comme incompatibles avec l'idéal de réhabilitation. Bien entendu, c'est dans le rapport Ouimet (1969) que l'on trouve la version la plus achevée de cette critique du pénitencier (encore au nom de la réhabilitation).

Donc, le grief le plus sérieux que nous pouvons faire à cet égard à la Commission c'est d'avoir mis en cause plutôt le principe de réhabilitation que le pénitencier lui-même ou encore les longues peines en tant que telles. Résultat: on continuera à condamner des personnes à des peines tout au moins aussi longues qu'à présent (p. 187), mais "sans le but" de les réhabiliter. Ce genre de critique à la théorie de la réhabilitation ne contribue ni à la modération ni à une plus grande efficacité du système.

Dès lors, que deviendra le pénitencier sans l'idéal de la réhabilitation? Le dilemme ici me semble être le suivant. D'un côté, la Commission a raison lorsqu'elle reproche au Medical model cette tendance à escamoter les aspects les plus punitifs de la sentence (d'incarcération) en présentant celle-ci comme une simple forme de traitement (p. 118). Cependant, de l'autre côté, il ne faut pas réduire la part d'une efficacité pratique incontestable de cet idéal dans les luttes et revendications internes (des détenus, de certains intervenants et agents d'unité, etc.) pour une amélioration des conditions de vie en prison et pour faire valoir, dans les décisions relatives aux détenus, une série des droits et des valeurs humaines et sociales. Il est difficile d'imaginer comment le pénitencier fonctionnera sans ce «drapeau» de la réhabilitation. Ne court-il pas le risque de succomber davantage à ces "pressions" qui ont tendance à considérer la "sécurité» comme un objectif en lui-même (rapport Swackhamer, 1972:36)? À mon avis, cette question réclame un examen et un débat plus approfondis. 
Car si la réhabilitation, tout au moins dans sa version la plus répandue, s'appuie sur des fausses prémisses (celle de l'anormalité du contrevenant); si elle mystifie aussi (ou plutôt mystifiait) le pénitencier en le présentant comme une sorte d' "hôpital», etc., elle introduit par contre un souffle humaniste que l'on ne retrouve en aucune autre théorie de la peine. Alors, à quoi bon bannir cet idéal, si l'on conserve intactes toutes les conditions matérielles qui produisent la souffrance et l' «anti-bienêtre» (C.R.D.C., 1975: 14) effectifs?

Pour conclure, je signale aussi que la Commission, lors de sa critique de la théorie de la réhabilitation (p. 139-142; 147-159), ne voit pas que cette théorie a été la seule théorie traditionnelle de la peine (utilitariste ou rétributiviste) à avoir préconisé - à proprement parler les mesures communautaires dans leur ensemble: elle est à l'origine du "sursis", de la probation, de tentatives pour revaloriser l'amende (au détriment de la prison), pour introduire le dédommagement, pour réduire les mesures punitives au-dessous des minimums prévus dans les codes afin d'atténuer les effets de stigmatisation, etc.

En effet, la revendication de ces «mesures communautaires et réparatrices" n'est pas un fait nouveau dans l'histoire des réformes pénales. Sans pouvoir entrer dans les détails d'un exposé analytique, disons que l'on trouve des éléments d'une telle démarche chez le magistrat français Bonneville de Marsangy (1864) et qu'elle fait aussi partie de la stratégie de réforme pénale préconisée et mise en valeur par l'École positive italienne (Garofalo, 1887; 1900), puis par le mouvement de défense sociale. C'est que l'utilitarisme pénal axé sur la réhabilitation revendique depuis son origine au XIXe siècle le recours à ces mesures pour diminuer les risques de récidive et/ou pour mieux réhabiliter.

La Commission attribue donc à tort au mouvement contemporain des victimes le monopole dans la revendication de ces mesures (p. 140). D'autre part, malgré toutes les ambiguïtés que cette théorie de la peine porte en elle, elle est la seule - toutes proportions gardées - à ne pas avoir fermé les portes aux mesures communautaires; elle est aussi la seule à ne pas concevoir le rôle du juge au moment de la détermination de la sanction comme fondamentalement d'ordre punitif. Et ce à tel point qu'on a voulu dans le passé attribuer cette tâche spécifique à d'autres groupes professionnels. 


\subsection{LES PROPOSITIONS DE RÉFORME ET LE PRINCIPE DE MODÉRATION}

Nous allons maintenant passer en revue quelques-unes des principales recommandations de réforme de la Commission (outre la déclaration du but de la sentence) compte tenu particulièrement du principe de modération. Nous avons retenu les points suivants:

i) la politique en matière de restrictions légales à la modération;

ii) la politique en matière de peines maximales;

iii) la sentence exceptionnelle;

iv) les cas de meurtre et de haute trahison;

v) les lignes directrices et la place donnée aux sanctions communautaires.

\subsection{LA POLITIQUE EN MATIÈRE DE RESTRICTIONS LÉGALES À LA MODÉRATION}

À l'heure actuelle, il existe dans le Code criminel trois types de dispositions législatives qui vont à l'encontre du pouvoir du juge d'octroyer une peine juste et appropriée aux caractéristiques de certains cas qui peuvent se présenter devant lui. Ces dispositions sont contraires au principe de la modération et ont été dénoncées comme une entrave au fonctionnement d'une «bonne justice» au moins depuis le rapport Archambault (1938):

a) les infractions à peine unique;

b) les infractions avec une peine minimale obligatoire;

c) les limites imposées à l'emploi des peines réparatrices ou communautaires.

Dans le premier cas, nous avons toutes les infractions dont la condamnation amène sans exception à une peine unique (maximale et minimale) à perpétuité. Présentement, elles sont au nombre de trois: la haute trahison (art. 47[1]), le meurtre au premier et au deuxième degré (art. 212/213 c.c.). Dans le deuxième cas, nous avons sept infractions (six dans le Code criminel, une dans la loi sur les stupéfiants) qui ont une peine minimale obligatoire d'amende ou d'emprisonnement. Elles sont définies par l'expression «au moins» ou équivalent. Exemple : emprisonnement «d'au moins sept ans» (p. 193-194). Enfin, dans le dernier cas, nous avons une disposition du Code criminel (art. 646[2]) qui empêche l'utilisation de l'amende lorsque l'accusé est déclaré coupable d'un acte criminel punissable d'un emprisonnement de plus de cinq ans (voir p. 411-412). 
Comme nous le verrons, la Commission ne se prononce pas sur les trois peines minimales à caractère unique, mais demande l'abolition totale des deux autres formes de restriction législative. Ce faisant, elle contribue d'autant plus de façon décisive à la mise en pratique du principe de modération que ces dispositions concernent des infractions relativement importantes, comme celle de la conduite avec des facultés affaiblies ${ }^{9}$.

\subsection{LES PEINES MAXIMALES ET LA LONGUEUR DES SENTENCES D'INCARCÉRATION}

Si nous ouvrons notre Code criminel et regardons les peines maximales pour chaque infraction, nous apprenons en réalité très peu sur la pratique actuelle des tribunaux. Comme la Commission l'a souligné à la suite de plusieurs autres documents, la structure des peines maximales de notre Code criminel n'a jamais été revue de façon exhaustive depuis 1892 et souffre de plusieurs anomalies (p. 214). Non seulement elles sont souvent exorbitantes (en elles-mêmes et/ou par rapport à la pratique), comme parfois elles ne reflètent plus une certaine perception sociale de l'échelle globale de gravité formelle des infractions.

La Commission proposera alors une restructuration et un ajustement dans les plafonds des maxima. Cependant, il est beaucoup plus difficile d'évaluer la signification de ces propositions par rapport au principe de modération. En effet, les peines maximales sont tellement éloignées de la pratique actuelle, que si les réductions proposées ne sont pas suffisamment marquées, elles ne contribueront ni à affecter la pratique actuelle ni à revaloriser de manière significative la "monnaie temps" (Rothman, 1981) dans le fonctionnement de la justice. Je crains que tel soit le cas ici. En regard des peines maximales, en effet, la Commission m'apparait moins concernée par le but de modération.

Le tableau 1 compare la structure actuelle des peines maximales (avec le nombre respectif d'infractions par catégorie) dans le Code criminel (C.cr.), Loi sur les stupéfiants (L.S.) et Loi des aliments et drogues (L.A.D.) avec la nouvelle proposition de la Commission.

9. La Cour Suprême du Canada vient de donner un appui indirect à ces recommandations de la Commission en considérant la peine minimale de 7 ans pour importation/exportation de drogue comme une peine "cruelle et inusitée" donc contraire à la Charte des droits et libertés de la personne. 
TABLEAU 1

La structure actuelle de peines et la proposition de la Commission

\begin{tabular}{llll}
\hline $\begin{array}{l}\text { Code criminel, } \\
\text { L.S. et L.A.D. }\end{array}$ & $\begin{array}{l}\text { Nombre/ } \\
\text { infractions }\end{array}$ & $\begin{array}{l}\text { Proposition de } \\
\text { la Commission }\end{array}$ & $\begin{array}{l}\text { Nombre/ } \\
\text { infractions }\end{array}$ \\
\hline $\begin{array}{l}\text { Perpétuité } \\
14 \text { ans }\end{array}$ & $\begin{array}{l}(32) \\
(50)\end{array}$ & $\begin{array}{l}\text { Perpétuité } \\
(3)^{*}\end{array}$ \\
10 ans & $(38)$ & 12 ans & $(17)$ \\
7 ans & $(3)$ & 9 ans & $(3$ \\
5 ans & $(87)$ & 6 ans & $(55)$ \\
3 ans & $(2)$ & 3 ans & $(78)$ \\
2 ans & $(86)$ & 1 an & $(71)$ \\
6 mois & $(65)$ & 6 mois & $(109)$ \\
3 mois & $(1)$ & & \\
Total & $(364)$ & & $(364)$ \\
\hline
\end{tabular}

Source: Ce tableau a été construit à partir des renseignements contenus dans l'annexe E du rapport de la C.C.D.P.; la haute trahison et le meurtre au premier et au deuxième degrés ont été ajoutés.

${ }^{*}$ La C.C.D.P. conserve la peine à perpétuité dans les cas de haute trahison et de meurtre au premier et deuxième degrés.

À première vue, les peines maximales ont été réduites drastiquement. De 32 infractions passibles d'un emprisonnement à perpétuité, il ne reste que trois, etc. En outre, si l'on exclut les trois infractions dont la Commission a décidé de ne pas traiter comme telles (la haute trahison et le meurtre au premier et deuxième degrés) (p. 228), la peine maximale dans le Code sera désormais de 12 ans plutôt que l'emprisonnement à perpétuitê. Globalement parlant, on constate aussi une tendance à la baisse à tous les niveaux.

Cependant, cette «réduction" dans l'échelle des peines est pour une grande partie plutôt illusoire. En effet, la peine maximale à perpétuité est tout simplement désuète d'un point de vue pratique dans la presque totalité de ces infractions. En outre, l'emprisonnement à perpétuité signifie présentement dans ces cas la possibilité de recevoir une libération conditionnelle après 7 ans.

D'autre part, en fonction des informations statistiques disponibles dans le rapport, je suis tenté de croire que ces modifications ne produiront pas non plus d'effets modérateurs sur la pratique, sauf peut-être pour les infractions dont la peine maximale sera d'au plus trois ans. Le tableau 2 
donne un aperçu du rapport entre les peines maximales actuelles, la pratique actuelle (telle que mesurée par la médiane et le $90^{\mathrm{e}}$ centile) et la peine maximale proposée par la Commission dans le cas de quelques infractions.

\section{TABLEAU 2}

Comparaison entre la pratique actuelle et les peines maximales proposées

\begin{tabular}{|c|c|c|c|}
\hline $\begin{array}{l}\text { Infractions/ } \\
\text { peines } \\
\text { actuelles }\end{array}$ & 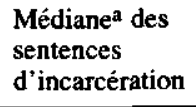 & $\begin{array}{l}90^{e} \text { centileb } \\
\text { des sentences } \\
\text { d'incarcération }\end{array}$ & $\begin{array}{l}\text { Peine } \\
\text { maximale } \\
\text { proposée }\end{array}$ \\
\hline $\begin{array}{l}\text { Hom. invol. coup. } \\
\text { (À perpétuité) }\end{array}$ & 5 ans & 12 ans & 12 ans \\
\hline $\begin{array}{l}\text { Tent. de meurtre } \\
\text { (À perpétuité) }\end{array}$ & 5 ans & 14 ans & 12 ans \\
\hline $\begin{array}{l}\text { Vol qualifié } \\
\text { (À perpétuité) }\end{array}$ & 2 ans & 7 ans & 9 ans \\
\hline $\begin{array}{l}\text { Introduction par } \\
\text { effraction } \\
\text { (À perpétuité ou } \\
14 \text { ans)c }\end{array}$ & 6 mois & 2 ans & 6 ans \\
\hline $\begin{array}{l}\text { Vol de plus de } \\
1000 \$ \\
(10 \text { ans) }\end{array}$ & 4 mois & 18 mois & 6 ans \\
\hline $\begin{array}{l}\text { Vol de carte de } \\
\text { crédit } \\
\text { (10 ans) }\end{array}$ & 3 mois & 1 an & 1 an \\
\hline $\begin{array}{l}\text { Voie de fait } \\
\text { simple } \\
(5 \text { ans })\end{array}$ & $1 \mathrm{mois}$ & 6 mois & 1 an \\
\hline
\end{tabular}

Source: Ce tableau a été construit à partir des données fournies par la C.C.D.P. (1987: $218 ; 225$ et annexe $F$ ).

a Médiane = sentence du milieu de l'échantillon; la moitié des sentences se situent au-dessus, l'autre moitié au-dessous.

b $90^{\circ}$ centile, soit $90 \%$ de l'ensemble des sentences d'incarcération se situent au-dessous.

c Maison d'habitation : à perpétuité; autres endroits: 14 ans.

Comme ces données l'indiquent, les peines maximales plus élevées (12 ans) coïncident approximativement avec le $90^{\mathrm{e}}$ centile. Si l'on considère en plus que la Commission recommande l'abolition de la libération conditionnelle, il y a peu de chances que ces nouvelles limites 
contribuent en tant que telles à la modération. La peine maximale proposée pour le vol qualifié dépasse "confortablement" le 90 e centile. Les peines maximales proposées dans les infractions contre la propriété sont dans certains cas trois fois plus élevées que le $90^{\mathrm{e}}$ centile actuel. Pour les infractions mineures (peine maximale d'au plus 1 an), les données disponibles suggèrent que les maxima sont plus ajustées à la pratique actuelle. Par ailleurs, ces infractions seront celles qui tomberont le plus massivement sous le coup des sanctions communautaires selon les lignes directrices proposées par la C.C.D.P.

Soulignons, en passant, que le plafond proposé comme point de départ pour structurer de manière décroissante toutes les peines d'emprisonnement demeure trop élevé si l'on le compare à ceux proposés par d'autres Comités dont la crédibilité demeure quand même hors de tout doute. Le Twentieth Century Fund Task Force (1976) propose que la limite supérieure des peines soit de huit ans; et von Hirsch (1976), dans son rapport Doing Justice, propose celle de cinq ans (voir C.C.D.P., p. 135, n. 13). La Commission signale, en outre, que la Commission de réforme du droit du Canada lui a proposé le plafond de sept ans comme hypothèse de travail (p. 135, n.13).

Comme la Commission semble le reconnaître, «les peines les plus sévères qui figurent dans nos propositions sont donc en fait très dures, aussi bien dans le système proposé qu'à la lumière de la pratique actuelle» (p. 224). La Commission d'ailleurs se garde prudemment de faire des références ici au principe de la modération et aux critiques séculaires adressées au pénitencier. Ceci dit, force est de reconnaître que même si les maxima proposées par la Commission demeurent trop élevées ${ }^{10}$, elles constituent un progrès par rapport à l'état actuel, contribuent à moderniser le Code criminel et à mieux hiérarchiser les infractions par rapport à l'échelle nominale adoptée.

\subsection{LA SENTENCE EXCEPTIONNELLE: UNE «ESCALADE» INATTENDUE}

Les propositions de la Commission à cet égard font durement contraste avec les critiques sur la prison dont elle fait état. En effet, la sentence «exceptionnelle» est une mesure particulièrement sévère qui vise à élargir encore plus les peines maximales de 9 et 12 ans. Elle vise, entre autres, à remplacer la "peine indéterminée» destinée au «délinquant

10. Les maxima auront des effets particulièrement néfastes sur le calcul de la asentence exceptionnelle» et de la "sentence globale» proposées par la Commission. Nous ne traiterons pas ici de ce dernier type de sentence (voir p. 244-246). 
dangereux" par une "peine de durée réelle». Cette mesure possède les caractéristiques suivantes:

- Toutes les infractions punissables de 9 et 12 ans peuvent être augmentées jusqu'à 50\% dans le cas d'une sentence exceptionnelle. Cela veut dire qu'une personne peut être condamnée à une peine allant jusqu'à 18 ans (i.e., 12 ans +6 ans). Il y aura 48 infractions susceptibles d'une telle peine (voir tableau 1).

- On élimine toute remise de peine et toute possibilité d'obtention d'une libération conditionnelle, même sur la partie de la peine qui a été ajoutée. Il suffit donc de condamner quelqu'un à une peine de 9 ans plus un jour pour durcir radicalement la peine.

Certes, la Commission indique quelques critères auxquels il faut répondre avant de demander une telle sentence et érige un certain nombre d'obstacles sur le plan de la procédure pour essayer de garder la nature "exceptionnelle» de la mesure (voir, p. 234-236). Sur le plan des critères, ils ne sont pas plus rassurants que ceux contenus dans les dispositions du même genre portant sur le «délinquant dangereux» et risquent de perpétuer le même problème de disparité dans l'application de la mesure (y compris celui d'une province à l'autre) déjà dénoncé par le rapport Ouimet (1969: 272)11. Les obstacles de procédure vont assurément réduire le recours à cette peine, mais ne vont pas empêcher une application discriminatoire de la loi.

La Commission semble croire qu'en éliminant le caractère «indéterminé» de cette peine, elle exorcise du même coup tous les maux qui l'entourent. Tel n'est pas mon avis. Certes, elle bouche une des «cheminées» du droit pénal qui permet un prolongement sans fin de la durée des peines. Mais elle la bouche assez haut et élargit son éventail d'applications possibles (48 infractions). Le prix que les détenus paieront ici pour avoir une «idée claire» du temps qu'ils passeront en prison est celui d'y rester avec certitude pour une longue période. Car cette sentence ne peut être révisée que suite à la fin de la période «ferme» de la sentence (i.e., 9

11. Un des critères retenus est celui selon lequel l'infraction dont l'accusé est déclaré coupable doit constituer un "sévice grave» contre la personne. Par ailleurs, on définit cette expression de manière très large et l'on renvoie à d'autres termes dont l'interprétation est fort subjective. En effet, les infractions impliquant des "sévices graves" sont celles «impliquant l'emploi ou la tentative d'emploi de la violence contre une autre personne, ou un comportement dangereux ou susceptible de l'être pour la vie ou la sécurité d'une autre personne. D'autre part, le comportement n'a pas besoin d'être de nature «brutalew; il suffit qu'il soit de nature "répétitive». Sur les problèmes de telles lois voir, entre autres, Price et Gold, 1976; Landreville et Petrunik, 1981; Jackson, 1982; Webster et Dickens, 1983. 
ou 12 ans dépendamment de la peine maximale de l'infraction pour laquelle ils ont été condamnés). Qui plus est, cette décision revient au même organisme qui les a condamnés pour la première fois. Ceci remet en question une des traditions au Canada qui est de ne pas investir un seul organisme de toute l'autorité relative à un domaine donnél2. En outre, je ne suis pas encore convaincu que la cour soit mieux placée que les commissaires des libérations conditionnelles du point de vue de leur connaissance du milieu carcéral et de tous les à-côtés sociaux entourant cette décision de révision. On pourrait, si l'on veut, prévoir un recours des détenus aux tribunaux en cas de refus du secteur correctionnel, mais jamais exclure complètement celui-ci d'un processus décisionnel de ce genre.

Une dernière remarque. La Commission soutient avoir "éliminé» la nature prédictive qui entoure normalement ces mesures (p. 232). Peutêtre. Mais d'une façon étrange. Un des critères possibles (mais non nécessaire) à l'application de cette mesure est que l'infraction doit être "de nature si brutale que l'on ne peut s'empêcher de conclure que le contrevenant constitue un danger pour la vie, la sécurité ou le bien-être physique des autres" (p. 234). La prédiction ici devient une présomption légale. À première vue, on abandonne les incertitudes de la prédiction scientifique pour la remplacer par la (l'apparente) «certitude" (à ne pas confondre avec «exactitude») d'une prédiction normative. Sur ce plan, on peut dire que le changement est plus formel que réel. Mais ajoutons à cela qu'il va falloir s'entendre sur le degré de gravité qui autorise (scientifiquement?) cette prédiction normative. On risque alors de réintroduire par la fenêtre ce que l'on a chassé par la porte: le débat sur le lien supposé entre les circonstances entourant le comportement passé et le comportement futur des personnes.

Peu importe leur titre (Loi sur le délinquant dangereux, mesures de sécurité ou sentence exceptionnelle), ce genre de mesure est incompatible avec le Justice Model et s'inspire directement de la théorie de la neutralisation du Medical Model. Mais depuis le XIX ${ }^{\mathfrak{e}}$ siècle, on n'a jamais réussi à imposer une seule logique au fonctionnement de la justice pénale. Le droit pénal est encore une forme de compromis -- où, bien entendu, les termes peuvent varier dans le temps et d'un pays à l' autre entre le droit pénal classique (Justice Model) et le droit pénal d'inspiration positiviste (Medical Model) (Tulkens et Digneffe, 1981). La Commission fait ici une concession à ce demier, même si elle essaie

12. La Commission reconnaît elle-même cette tradition et l'évoque pour justifier la création d'une commission permanente des sentences (voir p. 357). 
"d'habiller» sa proposition «à la mode du droit pénal classique». Ceci dit, la mesure aurait tout de même pu être moins musclée (peut-être au prix d'être un peu moins "habillée à la classique»). La remise de peine aurait pu être acceptée, la révision aurait pu être faite d'abord par la Commission des libérations conditionnelles, les délais auraient pu commencer à compter à partir du seuil supérieur prévu dans les lignes directrices (au lieu de la peine maximale), la durée maximale de la partie exceptionnelle pouvait se limiter à trois ans, etc.

Cette disposition est plus dure que la loi actuelle sur le «délinquant dangereux». Et il ne faut pas oublier que les peines peuvent déjà être prolongées en dépassant le seuil prévu dans les lignes directrices (jusqu'au maximum de la peine). Ces maxima constituent une large réserve de pouvoir et par rapport à la pratique et par rapport à toute concession raisonnable au principe de modération.

\subsection{LA COMMISSION FACE À L'IMPOSSIBLE PEINE: "UN QUART DE SIÈCLE MINIMUM"}

Comme nous le savons, la rançon que nous avons payée pour l'abolition de la peine de mort en 1976 a été celle d'accepter, en échange, une impossible peine: l'emprisonnement obligatoire pour un quart de siècle ( 25 ans) avant l'admissibilité à la libération conditionnelle. Cette peine s'applique au meurtre au premier degré et à la haute trahison, et peut également être appliquée (mais non nécessairement) dans le cas du meurtre au deuxième degré (p. 289). En gros, ces infractions sont susceptibles d'un emprisonnement à perpétuité, mais au lieu d'être éligibles à la libération conditionnelle après 10 ans, les condamnés doivent passer 25 ans en détention. Cette mesure a suscité - comme on s'en souviendra - un tollé de différents milieux; on invoquait alors des raisons qui pouvaient aller de son impossible gestion carcérale à sa contradiction flagrante avec les Droits de la personne (Landreville, 1985).

La Commission, quant à elle, a décidé de ne pas examiner les peines à perpétuité prévue pour ces infractions (p. 288). Si elle conserve ces peines, ce n'est pas qu'elle les trouve à propos, mais tout simplement qu'elle ne les a pas étudiées. Cependant, elle ne s'est pas tout à fait dérobée à la tâche et a décidé de revoir la question des délais préalables à l'admissibilité à la libération conditionnelle. Solution sans aucun doute partielle, mais conforme avec le principe de modération et avec l'importance croissante que nous accordons au Canada aux droits de la personne. 
Pour la haute trahison et le meurtre au premier degré, elle propose que le tribunal fixe la date d'éligibilité à la libération conditionnelle entre les limites de 15 et 25 ans; pour le meurtre au deuxième degré, les limites proposées sont respectivement de 10 et 15 ans. Pour certains au moins, la situation peut devenir quelque peu moins drastique, mais il vaut mieux se garder d'y voir une "bonne" solution à cette question.

\subsection{LES LIGNES DIRECTRICES ET LA PLACE DES SANCTIONS COMMUNAUTAIRES}

La Commission a décidé de proposer l'adoption de lignes directrices pour la détermination des sentences au Canada, aussi bien que la création d'une Commission permanente chargée, entre autres choses, de veiller à leur mise à jour et d'apporter les corrections nécessaires. Cette voie est d'autant plus intéressante qu'elle introduit une souplesse et un dynamisme importants au niveau de l'actualisation de la réforme pénale au plan des sanctions (p. 315-316).

Contrairement à ce que l'on pourrait penser de prime abord à partir de l'expérience américaine ${ }^{13}$, les lignes directrices n'ont pas été proposćes, chez nous, en vue de répondre essentiellement au problème de la disparité. Le modèle vise aussi à pouvoir donner une place (plus ou moins importante selon le cas) aux sanctions communautaires et à la modération.

Par rapport au "degré de contrainte" des lignes directrices, la Commission opte pour une formule intermédiaire: ni trop contraignante ni entièrement facultative. La Commission l'a désignée par "lignes directrices présomptives» (p. 332). Le terme présomption veut dire, entre autres choses, que si le juge décide de s"en écarter, il peut le faire. à condition de consigner par ecrit ses raisons de façon suffisamment explicite pour que leur bien-fondé puisse être èventuellement revu par la cour d'appel. C'est d'ailleurs la seule et unique contrainte qui pèse sur lui.

Soulignons, en passant, que la nécessité de disposer des lignes directrices présomptives mettant l'accent sur la réduction dans le recours à l'emprisonnement sera d'autant plus impérieuse que l'on décide d'abolir la liberation conditionnelle (p. 303-304).

13. Pour une analyse plus approfondie de la situation américaine. voir Brodeur ( 1985$)$. Ce travail aidera le lecteur à mieux comprendre les enjeux entourant la críation et l'élaboration des différents modèles de lignes directrites. 
Étant insatisfaite avec la forme des lignes directrices existantes (la "grille", les "points de référence", les "manuels", etc.), la Commission a décidé de bâtir un nouveau modèle auquel elle a donné le nom de "fiches types». Tel que conçu, ce système consiste d'abord à assortir toutes les infractions pénales d'une "présomption" qui sert à désigner d'une façon générale la nature de la sanction qui doit y prédominer (i.e., si elle doit être une sanction communautaire ou d'emprisonnement). Elle a fait cet exercice pour toutes les infractions du Code criminel, de la Loi sur les stupéfiants et de la Loi des aliments et drogues (parties III et IV). Ces présomptions sont au nombre de quatre. Je signale que j'ai modifié ici l'appellation officielle proposée par la Commission pour les deux premières $^{14}$ :

- Présomption de sanction communautaire sans réserve (SC);

- Présomption de sanction communautaire sous réserve ( $\mathrm{SCr}$ );

- Présomption d'emprisonnement sous réserve $(E r)$;

- Présomption d'emprisonnement sans réserve (E).

Comme on peut le constater, les présomptions ne désignent ni la sanction spécifique (par exemple, dédommagement, amende, etc.) ni le mode d'emprisonnement (e.g., emprisonnement discontinu, en milieu ouvert, etc.), mais plutôt la nature de la sanction. En outre, on voit que le principe de modération a joué un rôle dans la création de ces présomptions en ce sens que deux des quatre présomptions accordent une place absolue ou prioritaire aux sanctions communautaires.

Une fiche type peut porter soit sur une seule infraction (p. ex., une fiche pour l'homicide involontaire) soit encore sur un ensemble relativement homogène d'infractions (p. ex., le vol de plus de $1000 \$$, la fraude de plus de $1000 \$$, etc.). La Commission n'a eu le temps ni de préparer toutes les fiches, ni d'étudier suffisamment en détail la question des sanctions communautaires (SC). En réalité, elle n'a préparé que six fiches à titre d'illustration. Aucune de ces fiches ne porte sur la présomption de "sanction communautaire sans réserve". Le lecteur trouvera

14. La Commission a appelé ces présomptions comme suit: a) présomption de non-emprisonnement sans réserve (NE); b) présomption de non-emprisonnement sous réserve $(\mathrm{NEr}) ; c)$ présomption d'emprisonnement sous réserve $(\mathrm{Er}) ; d$ ) présomption d'emprisonnement sans réserve (E). Cette façon de désigner présente deux inconvénients. D'abord, elle définit toutes les présomptions exclusivement à partir de l'emprisonnement. Ceci est en contradiction avec certains propos de la Commission nous mettant en garde contre cette façon de concevoir les sanctions communautaires comme des simples «alternatives " à la prison (p. 85). Ensuite, les deux premières présomptions deviennent quelque peu rébarbatives, et tout particulièrement la première dont la compréhension n'est pas immédiate. 
en annexe un modèle simplifié des fiches. Le travail subséquent reviendra à la Commission permanente. Probablement parce que la question des SC n'a pas été approfondie, la seule fiche portant sur une présomption de $\mathrm{SCr}$ (p. 579) présente un problème particulier: elle est faite de la même façon que les autres. C'est-à-dire, les renseignements qu'elle donne au juge sur la pratique sentencielle actuelle porte exclusivement sur l'emprisonnement, et non sur les SC.

À l'heure actuelle, ces six fiches (de même que toutes les infractions examinées par la Commission) ont une caractéristique commune: elles ont chacune une seule sanction présomptive. Par ailleurs, les fiches peuvent prévoir différentes sous-catégories à l'intérieur de chaque infraction. C'est le cas de la fiche du vol qualifié (deux catégories). Ce qui change d'une catégorie à l'autre est le quantum de la sanction (i.e., les limites de la durée présomptive de l'emprisonnement) (voir annexe) ${ }^{15}$.

Examinons maintenant le sens des présomptions. Quels critères doivent, selon la Commission, régir le choix des présomptions? Elle en a retenu - à proprement parler - trois: la gravité formelle de l'infraction, la gravité interne de l'infraction et le casier judiciaire.

Le premier est le critère le plus important car il sert, à lui seul, à partager les infractions en deux grands groupes: les infractions recommandant un emprisonnement sans réserve (c'est-à-dire, même si l'infraction ne revêt pas de gravité interne et si l'inculpé n'a pas de casier judiciaire) et les infractions recommandant une sanction communautaire sans réserve (c'est-à-dire, même si l'inculpé a un long casier judiciaire).

Dans ce partage, il me semble que c'est le principe de proportionnalité (à connotation rétributiviste) qui a le rôle dominant, car c'est exclusivement en fonction de la gravité formelle des infractions qu'on a fait l'attribution des présomptions de base. Une fois identifiés les deux grands groupes extrêmes, on appliquera, semble-t-il, de façon prioritaire le principe de proportionnalité aux infractions situées en haut de l'échelle de gravité et le principe de modération à celles situées en bas de cette échelle.

15. À mon avis, le modèle est suffisamment souple pour pertnettre d'inclure éventuellement dans les fiches portant sur les infractions formellement plus graves (comme celle du vol qualifié) une double présomption pour rendre compte des formes moins graves ou encore relevant de circonstances particulières (on pourrait aussi, pour des raisons de clarté, préférer faire deux fiches, une pour chaque présomption). Ainsi, par exemple, supposons que la description du «vol qualifié $I$ » corresponde au vol à main armée dans une banque et que celle du «vol qualifié II", au vol de sac à main. On aurait pu prévoir pour ce dernier cas, et ce, sans trahir le modèle, une présomption de SC ou de SCr. Cependant, à l'heure actuelle, une telle possibilité n'a pas été envisagée. 
Outre ces deux groupes extrêmes d'infractions, la Commission a établi deux autres groupes (forcément intermédiaires) à partir d'une combinaison cumulative de deux autres critères: la gravité interne de l'infraction (i.e., par rapport aux autres formes que peut revêtir cette même infraction) (p. 343) et le casier judiciaire jugé pertinent ${ }^{16}$.

Cela veut dire concrètement que l'emprisonnement sous réserve (Er) signifie:

- que l'inculpé doit recevoir une sanction d'emprisonnement, à moins que les deux conditions soient présentes: l'infraction n'a pas pris de forme grave et l'inculpé n'a pas de casier judiciaire pertinent;

et que la sanction communautaire sous réserve $(\mathrm{SCr})$ signifie:

- que l'inculpé doit recevoir une sanction communautaire, à moins que les deux conditions soient présentes: l'infraction prend une forme particulièrement grave et l'inculpé a un casier judiciaire jugé pertinent.

Dans le premier cas, comme l'illustration I l'indique, il y a trois possibilités sur quatre qu'un résultat qui ne s'écarte pas de la présomption soit une sanction d'emprisonnement (en l'occurrence, les résultats B, C et $\mathrm{D})$.

Dans le deuxième cas, comme l'illustration suivante l'indique, c'est l'inverse qui se produit: il y a trois possibilités sur quatre que la décision conforme aux lignes directrices soit une SC. Comme on peut l'observer, la ligne noire divisant la «zone de l'emprisonnement» de la «zone des sanctions communautaires» se déplace de deux crans.

16. La définition de "casier judiciaire pertinent" n'est pas donnée. Il reviendra au tribunal de le définir (ou à la Commission permanente). Malheureusement, je ne peux pas faire ici la critique relative à l'adoption de ce critère. Il suffit de dire que la Commission n'en donne aucune justification socialement, éthiquement et même juridiquement recevables pour son adoption. Elle justifie ce critère tout simplement par la pratique actuelle. Implicitement, le message est le suivant: «le critère du casier judiciaire se justifie parce qu'on l'utilise présentement. La Commission tombe ici sous le coup de ce qu'on appelle, en éthique, la "guillotine de Hume" (Max Black). C'est-à-dire, cette manière habituelle et quasiment imperceptible de passer du constat d'un fait ( «les tribunaux adoptent ce critère") à un précepte normatif ou un jugement de valeur («les tribunaux doivent donc continuer à adopter ce critère»). Or, on ne peut pas passer sans plus du domaine de l' "être" à celui du «devoir être». Il faut déplorer le fait que la Commission n'ait pas profité de l'occasion pour attirer l'attention sur quelques problèmes relatifs à cette pratique. 


\section{ILLUSTRATION I}

Emprisonnement sous réserve (vol par effraction dans une maison d' habitation) (Er)

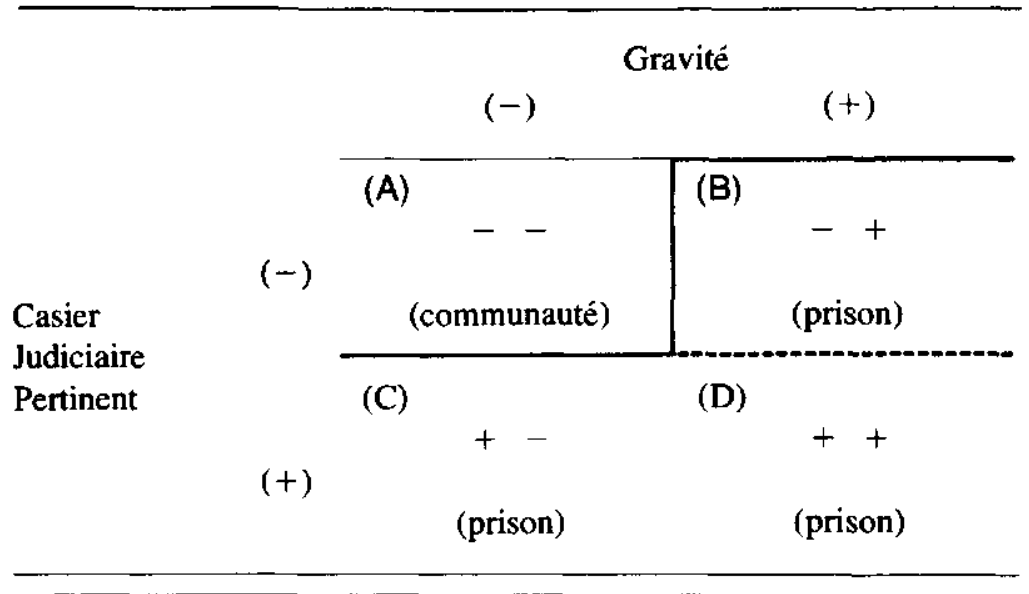

\section{ILLUSTRATION II}

Sanction communautaire sous réserve ( $\mathrm{SCr}$ )

(vol de plus de 1000\$)

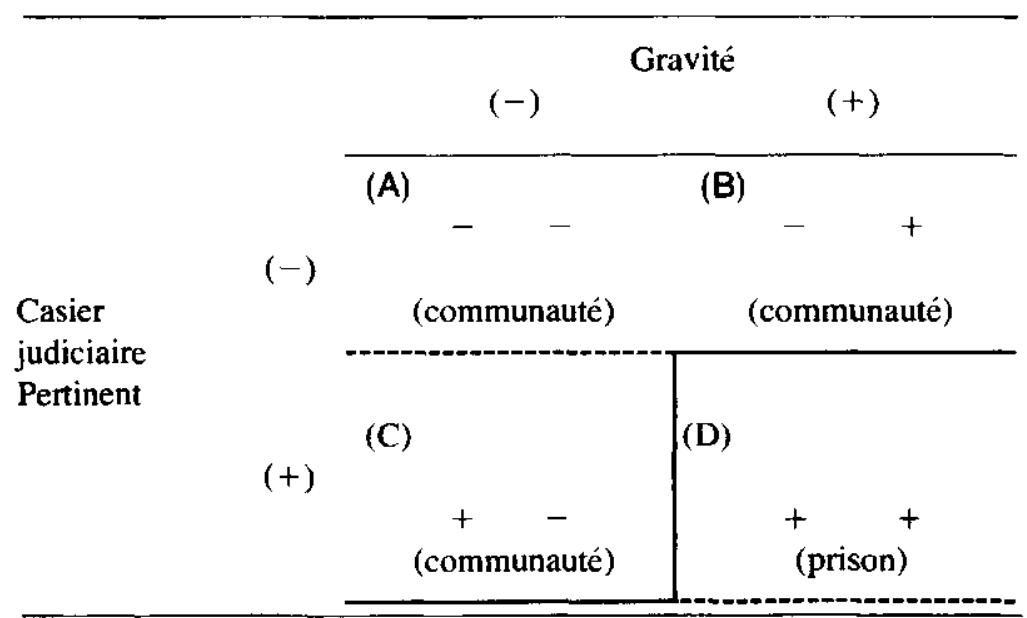

Le principe de la modération joue sans aucun doute un rôle important (par l'entremise du critère de la gravité interne) dans l'attribution des présomptions de base de ces infractions intermédiaires. Cependant, ce rôle a été neutralisé en très bonne partie par le critère (cumulatif) du casier judiciaire pertinent dans le cas d'infractions ayant reçu une présomption d'Er (parce que jugées formellement plus graves). 
Il faut examiner maintenant l'usage global de ces quatre présomptions par la Commission. D'abord, on constate que sur un total de 361 infractions (C.Cr., L.S., L.A.D.) les présomptions se distribuent grosso modo comme suit:

$$
\begin{array}{ll}
\mathrm{SC}=141(39 \%) & \mathrm{Er}=15(4 \%) \\
\mathrm{SCr}=137(38 \%) & \mathrm{E}=68(19 \%)
\end{array}
$$

La grande majorité des infractions ( $77 \%$ ) ont reçu une présomption favorisant les sanctions communautaires. Bien entendu, il faut garder en tête que ceci ne nous permet pas d'estimer précisément la portée effective de la mesure parce qu'il nous manque des renseignements statistiques sur le nombre de personnes annuellement condamnées pour ces infractions. Toutefois, une telle quantité d'infractions produira inévitablement (à supposer que les présomptions soient respectées) un certain impact sur le nombre d'admissions à la prison. D'autant plus que ces présomptions se rapportent massivement aux "petites infractions" qui sont justement celles qui occupent le plus le système. Le tableau 3 nous donne la distribution de ces présomptions par groupe d'infractions selon la peine maximale revue par la Commission.

Le tableau 3 nous permet de visualiser, entre autres choses, l'orientation qu'a prise la réforme proposée par la Commission. Elle consiste fondamentalement en une réduction du recours à l'emprisonnement dans le cas des infractions moins graves (petites infractions contre la propriété, l'ordre public, etc.) et à maintenir - voire resserrer - l'usage actuel de l'emprisonnement pour les infractions formellement plus graves. On peut voir qu'au fur et à mesure que l'on descend dans le tableau, les chiffres se déplacent aussi vers les peines d'emprisonnement. Les infractions ayant une peine maximale de trois ans semblent faire la démarcation entre les deux groupes.

Les infractions de six ans sont divisées en deux blocs, mais sans étudier statistiquement le poids de chaque infraction, il est difficile d'estimer de quel côté la balance va pencher. À ce propos, il ne faut pas oublier que la Commission a malheureusement recommandé une présomption d'Er pour l'introduction par effraction dans une maison d'habitation (peine maximale : 6 ans). Or, même si les données statistiques ne nous permettent pas généralement de distinguer entre l'introduction par effraction dans une maison d'habitation et dans un autre lieu, il n'en demeure pas moins que cette infraction, dans son ensemble, rend compte du deuxième plus haut pourcentage d'adultes du sexe masculin mis en accusation par la police dans la catégorie de crimes contre la propriété (Billingsley, 1983: 2). 
TABLEAU 3

La fréquence des présomptions par groupe d'infractions selon la peine maximale

\begin{tabular}{|c|c|c|}
\hline Peines maximales & N. d'infractions & Présomptions \\
\hline 6 mois & 109 & $\begin{aligned} \mathrm{SC} & =99 \\
\mathrm{SCr} & =10 \\
\mathrm{Er} & =0 \\
\mathbf{E} & =0\end{aligned}$ \\
\hline 1 an & 71 & $\begin{aligned} S C & =42 \\
S C r & =28 \\
E r & =1 \\
E & =0\end{aligned}$ \\
\hline 3 ans & 78 & $\begin{aligned} \mathrm{SC} & =0 \\
\mathrm{SCr} & =69 \\
\mathrm{Er} & =6 \\
\mathrm{E} & =3\end{aligned}$ \\
\hline 6 ans & 55 & $\begin{aligned} S C & =0 \\
S C r & =29 \\
E r & =8 \\
E & =18\end{aligned}$ \\
\hline 9 ans & 31 & $\begin{array}{c}S C=0 \\
S C r=1 \\
E r=0 \\
E=30\end{array}$ \\
\hline 12 ans & 17 & $\begin{array}{c}S C=0 \\
S C r=0 \\
E r=0 \\
E=17\end{array}$ \\
\hline
\end{tabular}

Finalement, pour quelques-unes des infractions qui se situent dans la partie inférieure du tableau, je crains qu'il y ait un risque non négligeable d'augmentation dans la longueur de l'emprisonnement si la libération conditionnelle est abolie et si la remise de peine est réduite à un quart. Bien entendu, ce risque dépendra également du comportement des juges dans l'octroi de peines. Il faut espérer qu'ils réduiront la longueur des sentences. Une analyse plus précise de cette orientation ne saurait se faire sans le support des données statistiques.

\subsection{CONCLUSION: UN BILAN PROVISOIRE CONCERNANT LA MODÉRATION}

Tout compte fait, les propositions de réforme de la Commission favorisent-elles ou non la modération? La question ainsi libellée est 
difficile — voire impossible — à répondre. Il faut regarder séparément différents aspects de la question.

Examinons d'abord particulièrement les propositions présentées dans la section précédente.

En ce qui concerne la fréquence du recours à l'emprisonnement dans le cas des infractions de 3 ans et moins, la réponse me paraît hors de tout doute affirmative. À cet égard, la Commission a fait réellement beaucoup et ce n'est pas la moindre des tâches. Si l'on prend au sérieux ces propositions, je crois qu'on réussira à introduire définitivement dans les "moeurs pénales" les sanctions communautaires et réparatrices jusqu'ici qualifiées tout au plus d' "alternatives" à l'incarcération.

En ce qui concerne la longueur des peines, là justement où la modération fait le plus cruellement défaut (chez les infractions passibles d'une peine de 6 ans et plus), force est de constater que la Commission n'introduit aucun progrès particulièrement visible. Sans pouvoir être incisif, je dirais que la Commission a plutôt adopté à cet égard une politique de stabilisation, combinée, ici et là, avec des traits de durcissement assez apparents. La modération n'a pas eu grand mot à dire ici en dépit du fait que le rapport introduit avec tambour et trompettes les critiques séculaires faites au pénitencier. C'est que ces critiques ont été de toute évidence entièrement consommées dans l'effort pour déplacer le recours aux courtes peines d'incarcération (afin de créer un espace pour les $\mathrm{SC}$ ) et pour déloger de force le but de "réadaptation» de l'avant-scène du droit pénal. Ironiquement, les critiques historiquement adressées particulièrement au pénitencier et aux longues peines ont produit des effets positifs surtout sur la prison et les courtes peines. Ironiquement aussi, les critiques au pénitencier ont servi à démontrer que le pénitencier «ne réhabilite pas», mais du même coup l'ont rescapé indemne de la tempête, prêt à servir encore une fois fidèlement des «nouveaux" objectifs (lesquels?), sans avoir subi aucune transformation notable. À ce propos, certaines parties du rapport m'ont donné l'impression de véhiculer le message suivant: le problème du pénitencier est tout simplement celui de vouloir "réadapter".

D'autre part, soulignons que si l'on applique aux longues peines d'emprisonnement la critique (essentiellement juste) que la Commission adresse à toutes les théories de la peine (p. 147-158), on aboutit à une constatation surprenante: le pénitencier et les longues peines ne trouvent aujourd' hui - dans la meilleure des hypothèses - que des justifications très fragiles. En effet, au nom de quoi doit-on incarcérer quelqu'un pour neuf, douze, dix-huit ( sentence exceptionnelle») ou, pire encore, vingt- 
cinq ans? Ce ne serait assurément pas pour les «réhabiliter» (p. 151-153); ce ne serait pas non plus ni pour dissuader ni pour réduire le taux de criminalité par neutralisation (selon ce qui découle de l'analyse que la Commission a faite des recherches récentes, p. 148-151; 153-155); l'objectif de dénonciation, quant à lui, ne réclame pas des peines aussi élevées et la théorie du juste dû s'oppose à de telles peines. Pourquoi, alors? Pour "rétribuer le mal par le mal»? Peut-être, mais comme le souligne la Commission, cette réponse est "très peu instructive» (p. 155) et le rétributivisme constitue une justification fort problématique de la peine (p. 156). Peut-on dire alors que les longues peines se justifient par "un quelconque besoin d'assouvir une pulsion vindicative de la part de l'opinion publique"? Tel n'est pas non plus le point de vue de la Commission. Même si l'on suppose que tel soit effectivement le vou de l' "opinion publique», le Droit ne saurait considérer un tel argument comme étant, à lui seul, recevable sans avoir à renoncer du même coup à toute recherche de Justice (ou à se prononcer sur des phénomènes comme le racisme ou le sexisme jà où la "majorité" le soutient, etc.). Si l'on cherche la réponse dans la nouvelle théorie de la sentence de la Commission, on pourrait être tenté de voir dans le principe de proportionnalité la justification de telles peines. Mais ce n'est pas le cas. La Commission reconnaît qu'il n'existe pas de principes théoriques ni de critères intrinsèques permettant de justifier comme tel un certain plafond des maxima (p. 224). Pour le faire, elle décide alors de se référer à la pratique actuelle (p. 220). Dommage que la pratique ne soit pas non plus à elle seule une justification philosophiquement recevable (voir note n.16). Qui plus est, Brodeur (1986: 165-169) démontre de manière convaincante que le principe de proportionnalité ne sert ni à justifier ni à opérationnaliser le quantum des peines. Résultat: on est poussé à conclure que si J'on continue à octroyer des longues peines, c'est simplement parce que cela fait partie des "mœurs" (dont le bien-fondé ne fait pas l'objet d'examen ni de révision).

Cette remarque m'amène à un deuxième ordre de considérations.

Il me semble que la Commission a adopté un procédé de réforme qui est à certains égards apparenté à celui préconisé par la théorie du juste dû ${ }^{17}$. En gros, ce procédé, dans la théorie du juste dû, consiste à situer toutes les infractions dans une échelle ordinale allant de la plus (formellement) grave à la moins grave. Ensuite, on assortit ces infractions de "punitions" (la Commission dirait «sanctions") qui sont censées réfléter la gravité relative de la conduite telle que formellement décrite. C'est le principe de la proportionnalité (formelle) des infractions qui guide cette

17. Voir à ce propos tout particulièrement von Hirsch (1986: chap. 4). 
opération de hiérarchisation. Aucun autre critère n'est utilisé pour préciser le contenu ou le contexte de ces infractions.

Un des problèmes de ce procédé (et des énoncés théoriques du modèle) est qu'il devient formellement sélectif dans I'application du principe de modération : la modération a tendance à se concentrer vers le «bas de l'écheile». Comment cela se produit-il?

Compte tenu de l'importance exagérée que la théorie du juste dû accorde au principe de proportionnalité et à la dimension formelle des infractions, elle fait fi du principe de la mesure appropriée la plus adéquate et ne conçoit la modération que de deux manières: soit par rapport aux infractions mineures et plus banales, soit encore par le biais de la réduction de l'ensemble de l'échelle globale des peines. On essaie alors de «baisser» désespérément les peines maximales, parce que c'est la seule façon par laquelle ce modèle (cette théorie) permet au principe de modération de toucher aux infractions plus graves. Von Hirsch (1976) va alors proposer que la peine maximale soit de 5 ans. Car, en cas contraire, son modèle sera toujours assez sauvage envers les infractions du haut de l'échelle. Mais, cette recommandation est aussi de toute évidence la plus difficile à réaliser politiquement parlant. La théorie du juste dû présente donc des limites importantes non seulement du point de vue théorique, mais aussi stratégique et pratique. Le principe de modération prend donc une acceptation et une mise en forme trop étroites.

Or, ce problème est d'autant plus important que les infractions formellement mineures sont, toutes proportions gardées, toujours «réellement mineures». Sans mystifier, il n'y a pas beaucoup de manières de "transformer" le vol à l'étalage en quelque chose de "réellement» très grave. Les petites infractions, lorsqu'elles deviennent réellement graves, se transforment en d'autres infractions (le chef d'accusation change). Certes, on peut fort bien concevoir qu'un vol à l'étalage réalisé dans le cadre d'un présumé "réseau de vols" puisse être considéré moins banal que les autres vols de cette catégorie. Mais cette forme "plus grave» trouve vite en elle-même ses propres limites de gravité réelle (par rapport au «dommage» en soi-même et par rapport aux autres infractions ou formes de dommage légalement tolérées).

Tel n'est pas le cas des infractions formellement graves. Celles-ci ont d'abord la particularité de provoquer l'imagination et d'évoquer à sa simple désignation (e.g., "homicide involontaire coupable», «inceste», voies de fait grave, vol qualifié, introduction par effraction, etc.) les aspects du problème qui nous répugnent ou effraient le plus. Par ailleurs, ces infractions formellement graves, contrairement aux infractions mi- 
neures, désignent des réalités fort hétérogènes, des situations humainement fort complexes et ayant lieu dans des contextes fort différents. Les définitions légales sont ici, pour ainsi dire, "heuristiques" en ce sens qu'elles sont là pour couvrir (en théorie) le plus grand nombre de "faits-significations" possibles; bref, elles peuvent abriter une diversité interne d'autant plus réellement significative que celle-ci risque fort de contraster avec nos suppositions imaginaires et/ou parti-pris moralistes. C'est donc au niveau des infractions formellement graves que nous trouvons les "simplifications» les plus abusives de la typification pénale (le problème du «nominalisme juridique»). D'où le piège de ce procédé : là justement où on a besoin d'être le plus attentif et nuancé pour répondre de façon appropriée aux particularités du cas, c'est là aussi où on a le plus tendance à court-circuiter, à mettre tout dans le même (mauvais) paquet, etc.

Or, on reconnaît aujourd'hui de plus en plus que le droit pénal est un instrument très néfaste et clairement inadéquat tout au moins pour traiter d'un grand nombre de situations problèmes complexes qui historiquement sont passées sous son emprise (Becker, 1975: passin; Mohr, 1980: 25; Dumont, 1986: passin; Landreville, Blankevoort et Pires, 1981: 39-41, 145, 161-162; Pires et Vallières, 1986: 91-96). Tel est le cas, par exemple, des "crimes sans victime", mais aussi de conflits et agressions (parfois très graves) impliquant des gens qui ont une relation soutenue, comme les problèmes en milieu familial, en milieu de travail, entre voisins ou encore dans certains cas de bagarres et conflits entre jeunes adultes pour ne mentionner que les plus connus. Bien entendu, cette façon de poser la question ne couvre pas toutes les dimensions du problème posé par la diversité interne de certaines infractions formellement plus graves. Mais elle visualise les points les plus dramatiques. Le problème est que toute cette question de l'inopportunité du droit pénal pour faire face à certaines situations problèmes complexes et/ou hétérogènes n'est ni posée ni discutée par le juste dû (et par cette conception de la modération). On oublie que le droit pénal et la prison sont - pour emprunter une expression de Dumont (1986: 28) - un "instrument massue» fort inapproprié à certains problèmes (graves).

La Commission reste partiellement prise au même piège. Car elle ne réussit pas à réduire vraiment le point de départ de l'échelle punitive, ni à faire "remonter» explicitement jusqu'aux infractions ayant une présomption d'emprisonnement $(E=68 ; E r=15)$ le principe de la sentence appropriée la moins lourde. Il semble bien que ce soit le principe de la proportionnalité qui prend essentiellement la relève ici. Une autre façon d'approcher le problème aurait pu permettre (en l'absence d'obstacles de 
principe) à la Commission d'étendre le principe de la sentence appropriée à d'autres infractions formellement graves et d'adapter les fiches types et les présomptions en conformité.

Par ailleurs, elle intègre bel et bien le principe de la sanction appropriée la moins lourde dans sa Déclaration du but de la sentence et le met en application effective et directe par rapport à un grand nombre d'infractions. Elle se défait brillamment du monopole absolu de la notion de punition et de peine au niveau de la pratique sentencielle. Sa nouvelle théorie de la sentence ouvre des voies particulièrement prometteuses. Par conséquent, elle provoque une extension remarquable des sanctions communautaires. Mais ce n'est pas tout. Elle met sur pied un modèle fort ingénieux de lignes directrices, écartant - jusqu'où il nous est donné de voir à l'étape actuelle - maints problèmes et erreurs commis par les expériences pionnières américaines. Son modèle a l'avantage supplémentaire non négligeable d'être suffisamment flexible pour pouvoir s'adapter, si l'on veut, au principe de la modération dans le cas des infractions qui ont été pour l'instant délaissées. La "tension" consacrée par la Déclaration du but de la sentence entre ce principe et celui de la proportionnalité n'est pas anéantie pour autant et l'enjeu va dépendre maintenant en partie des tribunaux, en partie de la Commission permanente. La «sanction juste» dépendra alors entièrement de la volonté personnelle du juge (et/ou du procureur de la couronne dans le cas d'une recommandation) de s'écarter, dans certains cas, des présomptions de base. Bref, il y a de très bonnes cartes sur table; quelques pièges demeurent encore; pour le reste, c'est partie remise.

\section{RÉFÉRENCES}

AMERICAN FRIENDS SERVICE COMMITTEE, Struggle for Justice, New York, Hill \& Wang, 1971.

ARCHAMBAULT, RAPPORT, Rapport de la Commission royale d'enquête sur le système pénal du Canada, Ottawa, Imprimeur de Sa Très Excellence Majesté le roi, 1938.

BECKER, C. "La résolution des conflits et le recours aux tribunaux", dans la Commission de réforme du droit du Canada: Études sur la déjudiciarisation, Ottawa, Information Canada, 1945.

BÉLIVEAU, P. et J. PRADEL, la Justice pénale dans les droits canadiens et français, Montréal, Les Éditions Yvon Blais Inc., Paris, Cujas, 1986.

BILLINGSLEY, B., «Empirical Sentencing Data Project: Report \# 3, Break and Enter», dans Research Group, Sentencing Practices and Trends in Canada, Ottawa, A serie of reports prepared between the Department of Justice Canada and the Research Group, 1983. 
BONNEVILLE de MARSANGY, A., De l'amélioration de la loi criminelle, vol. I et II. Paris, Cosse et Marchal, 1864.

BRODEUR, J.P., "Réforme pénale et sentences: expériences nord-américaines", Déviance et Société, 9, (3), 1985, pp. 165-200.

BRODEUR, J.P., «Provocations», Criminologie. 19, 1986. pp. 141-169.

CANADA, GOUVERNEMENT, le Droit pénal dans la société contemporaine, Ottawa, gouvernement du Canada, 1982.

CANADA, GOUVERNEMENT, la Détermination de la peine, Ottawa, gouvemement du Canada, 1984.

COMMISSION CANADIENNE SUR LA DÉTERMINATION DE LA PEINE, Réformer la sentence : une approche canadienne, Rapport de la Commission canadienne sur la détermination de la peine (C.C.D.P.). Ottawa, ministre des Approvisionnements et Services Canada, 1987.

COMMISSION DE RÉFORME DU DROIT DU CANADA, Bientôt là..., Quatrième rapport annuel 1974-1975, Ottawa, Information Canada, 1975.

DECORE, J.V., "Criminal Sentencing: The Role of the Canadian Courts of Appeal and the Concept of Uniformity", Criminal Law Quarterly, 6. 1964, pp. 324-380.

DUMONT, H., le Contrôle judiciaire de la criminalité familiale, Montréal, Les Éditions Thémis, 1986.

FAUTEUX, RAPPORT, Comité institué pour faire enquête sur les principes et les méthodes suivis au service des pardons du ministère de la Justice du Canada, Ottawa, Imprimeur de la reine, 1956.

GAROFALO, R., Riparazione alle vittime del delitso, Turin, Bocca, 1887.

GarofalO, R., Criminology, N.J., Patterson-Smith, 1914, pp. 419-435.

GOFFMAN, E., Asiles, Paris, Minuit, 1968.

HIRSCH, A. von, Doing Justice: The Choice of Punishment, New York, Hill et Wang, 1976.

HIRSCH, A. von, Past and Future Crimes - Deservedness and Dangerousness in the Sentencing of Criminals, New Brunswick, Rutgers University Press, 1985.

HOGARTH, J., Sentencing as a Human Process. Toronto, University of Toronto Press, 1971.

JACKSON, M., Sentences that Never End: The Report on the Habitual Criminal Study, Vancouver, Université de la Colombie Britannique, décembre 1982.

LANDREVILLE, $P$. et M. PETRUNIK, "Le "délinquant dangereux" dans les législations nord-américaines », dans Ch. Debuyst (édit.), Dangerosité et Justice pénale : ambiguïté d'une pratique, Genève, Masson, 1981, pp. 207-229.

LANDREVIL.LE, P., V. BLANKEVOORT, A.P. PIRES, les Coùts sociaux du système pénal, Ottawa, Rapport de recherche à l'intention du ministère du Solliciteur général du Canada, 1981.

LANDREVILLE, P., Vingt-cinq ans d'emprisonnement : châtiment efficace et humain?. Communication prononcée au Congrès de l'Association canadienne de la justice pénale, Vancouver, du 7 au 11 juillet 1985. 
LANDREVILLE, P., «Surveiller et prévenir: l'assignation à donicile sous surveillance électronique ", Déviance et Société, 11 , (3) (à paraître, 1987).

MOHR, J.W., "New Directions in Sentencing», dans B.A. Grossman (édit.), New Directions in Sentencing. Toronto, Butterworths, 1980.

OUIMET, RAPPORT, Rapport du Comité canadien de la réforme pénale et correctionnelle. Justice pénale et correction: un lien à forger, Ottawa, Imprimeur de la reine, 1969.

PIRES, A.P., Inventaire d' extraits significatifs ayant trait à la disparité des sentences dans la littérature canadienne, Ottawa, Étude préparée pour la Commission canadienne sur la détermination de la peine, 1984.

PIRES, A.P., Critiques à la prison et principe de modération: inventaire d' extraits dans les documents canadiens, Ottawa, Étude préparée pour la Cornmission canadienne sur la détermination de la peine, 1985 .

PIRES, A.P. et S. VALLIÈRES, Droits de la personne et information juridique: une recherche évaluative. Ottawa, Direction de la planification et de l'élaboration de la politique, Section de la recherche et de la statistique, ministère de la Justice du Canada, 1986.

PRICE, R.R. et A.D. GOLD, "La surveillance judiciaire du délinquant dangereux", dans la Commission de réforme du droit du Canada, Études sur l'emprisonnement, Ottawa, ministère des Approvisionnements et Services Canada, 1976, pp. 163-261 .

QUÉBEC, GOUVERNEMENT, Rapport du Comité d'étude sur les solutions de rechange à l'incarcération, Québec, ministère du Solliciteur général du Québec, 1986.

ROTHMAN, D.J., "Doing Time: Days, Months and Years in the Criminal Justice System», dans H. Gross et A. von Hirsch (édit.), Sentencing, N.Y., Oxford University Press, 1981, pp. 374-385.

SWACKHAMER, REPORT, Report of the Commission of Inquiry into certain Disturbances at the Kingston Penitentiary during April 1971, Ottawa, Sollicitor General, Canada, 1972.

TULKENS, F. et F. DIGNEFFE, «La notion de dangerosité dans la politique criminelle en Europe occidentale", dans Ch. Debuyst (édit.), Dangerosité et justice pénale : ambiguitte d'une pratique. Genève, Masson, 1981, pp. 191-205.

TWENTHIETH CENTURY FUND TASK FORCE ON CRIMINAL SENTENCING, Fair and Certain Punishment, New York, McGraw-Hill, 1976.

WEBSTER, C., B. DICKENS, la Détermination de l'état dangereux: pour une nouvelle politique à l'égard des délinquants dangereux, Étude préparée à l'intention du ministère de la Justice du Canada, 1983. 


\section{ANNEXE}

Modèle simplifié d' une fiche type

\begin{tabular}{|c|c|c|c|}
\hline I & Infraction: & \multicolumn{2}{|l|}{$\begin{array}{l}\text { Vol qualifié } \\
\text { (art...) }\end{array}$} \\
\hline II. & Peine maximale: & \multicolumn{2}{|l|}{9 ans } \\
\hline III. & Sentence présomptive: & \multicolumn{2}{|c|}{ Emprisonnement (E) } \\
\hline \multirow[t]{4}{*}{ IV. } & Durée présomptive: & & \\
\hline & Catégorie & Description & Durée \\
\hline & Vol qualifié I: & $\circ \ldots . . . . \$$ & 2 à 4 ans \\
\hline & Vol qualifié II: & ${ }^{\circ} \ldots \ldots \ldots \S$ & 4 à 16 mois \\
\hline \multirow[t]{2}{*}{ v. } & \multicolumn{3}{|l|}{ Pratique actuelle: } \\
\hline & \multicolumn{3}{|c|}{$\begin{array}{l}\text { 'On donne ici des renseignements sur la longueur des sentences d'emprisonne- } \\
\text { ment§ }\end{array}$} \\
\hline VI. & \multicolumn{3}{|l|}{$\begin{array}{l}\text { Jurisprudence: } \\
{ }^{\circ} \text { Extraits de jugements\$ }\end{array}$} \\
\hline
\end{tabular}

